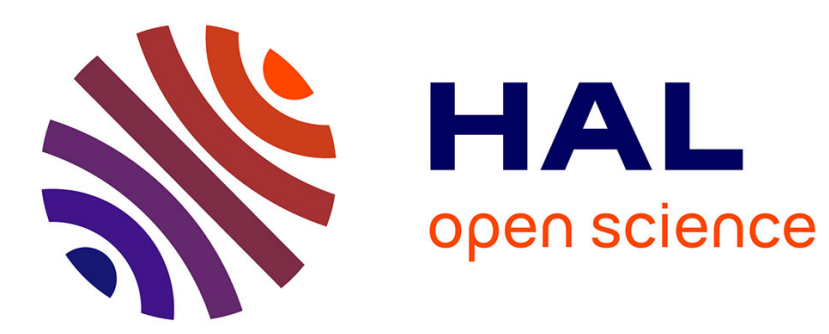

\title{
Aerodynamic analysis and optimization of a boxwing architecture for commercial airplanes
}

Marco Carini, Michaël Méheut, Stylianos Kanellopoulos, Vittorio Cipolla, Karim Abu Salem

\section{- To cite this version:}

Marco Carini, Michaël Méheut, Stylianos Kanellopoulos, Vittorio Cipolla, Karim Abu Salem. Aerodynamic analysis and optimization of a boxwing architecture for commercial airplanes. AIAA SCITECH 2020, Jan 2020, ORLANDO, United States. 10.2514/6.2020-1285 . hal-02904536

\section{HAL Id: hal-02904536 \\ https://hal.science/hal-02904536}

Submitted on 22 Jul 2020

HAL is a multi-disciplinary open access archive for the deposit and dissemination of scientific research documents, whether they are published or not. The documents may come from teaching and research institutions in France or abroad, or from public or private research centers.
L'archive ouverte pluridisciplinaire HAL, est destinée au dépôt et à la diffusion de documents scientifiques de niveau recherche, publiés ou non, émanant des établissements d'enseignement et de recherche français ou étrangers, des laboratoires publics ou privés. 


\title{
Aerodynamic analysis and optimization of a boxwing architecture for commercial airplanes
}

\author{
M. Carini ${ }^{1}$, M. Meheut ${ }^{2}$, S. Kanellopoulos ${ }^{3}$, \\ ONERA The French Aerospace Lab, Meudon, F-92140, France \\ V. Cipolla ${ }^{4}$ and K. Abu Salem
University of Pisa, Via G. Caruso 8, Pisa, Italy
}

In the present work, the CFD analysis and local shape optimization of a boxwing architecture designed during the early stages of the PARSIFAL project are addressed in order to assess and improve its transonic aerodynamic performance. The assessment of the baseline configuration is carried out by means of highfidelity RANS computations while an Euler-based workflow is employed for the optimization study. In both cases, CFD computations are supplemented by a far-field drag post-processing to inspect the behavior and the impact of the different drag sources (induced, wave, pressure and viscous dissipation) on the aerodynamic performance. Results obtained from the optimization of local twist and camber parameters for the isolated boxwing lifting-system are presented and discussed. The optimization successfully achieves a great reduction of the compressibility effects affecting the baseline configuration, leading to a substantial improvement of the aerodynamic performance at cruise and higher values of the lift coefficient.

\section{Nomenclature}

$\begin{array}{ll}M & =\text { Mach number } \\ R e & =\text { Reynolds number } \\ A o A & =\text { angle of attack } \\ C p & =\text { pressure coefficient } \\ C L & =\text { lift coefficient } \\ C D & =\text { drag coefficient } \\ C D_{p} & =\text { pressure drag coefficient } \\ C D_{f} & =\text { friction drag coefficient } \\ C D_{i} & =\text { induced drag coefficient } \\ C D_{v p} & =\text { viscous pressure drag coefficient } \\ C D_{w} & =\text { wave drag coefficient } \\ e & =\text { Oswald efficiency }\end{array}$

\section{Introduction}

The project PARSIFAL (Prandtlplane ARchitecture for the Sustainable Improvement of Future AirpLanes) [1], with a consortium made of 6 partners from 4 different European countries (University of Pisa, University of Delft, ONERA, DLR, ENSAM, SkyBox Engineering S.r.l.), aims at defining the basis to improve the future air transport

\footnotetext{
${ }^{1}$ Dr., Research Engineer, Aerodynamics, Aeroelasticity and Acoustics Dept., marco.carini@onera.fr.

${ }^{2}$ Dr., Senior Research Engineer, Aerodynamics, Aeroelasticity and Acoustics Dept., michael.meheut @onera.fr.

${ }^{3}$ Intern, Aerodynamics, Aeroelasticity and Acoustics Dept., stylianos.kanellopoulos@onera.fr.

${ }^{4}$ Assistant Professor, Department of Civil and Industrial Engineering/Aerospace Section, vittorio.cipolla@ unipi.it.

${ }^{5} \mathrm{PhD}$ Student, Department of Civil and Industrial Engineering/Aerospace Section: karim.abusalem@gmail.com.
} 
by evaluating the effects of introducing the innovative boxwing aircraft [3-5], called "PrandtlPlane" $(\operatorname{PrP})$, into service. The project is focused on the medium size commercial aircraft category. The adoption of the $\operatorname{PrP}$ configuration for this category can confer to an aircraft with the same overall dimensions and fuel consumption of an A320/B737 the payload capacity of an A330/B767. This can strongly contribute to improve the air traffic in all the airports, including the ICAO Aerodrome Reference Code $\mathrm{C}$ ones.

From theoretical viewpoint, it is well known that the boxwing configuration presents the minimum induced drag among all possible wing architectures, as shown by Prandtl in 1924 [2] and proved by Frediani [3] and Frediani and Montanari [4]. Notwithstanding, the boxwing architecture can offer several additional advantages w.r.t. the traditional "tube and wing" configuration concerning maneuvering, engine integration, structure design and operability. As an example, the stall speed is lower than any equivalent monoplane and the stall onset is usually very smooth. Pitch control can act as a pure couple without modifying the total lift during maneuvers. Many engine integration solutions are possible and aeroelastic effects can be controlled without great weight penalties, due to the over-constrained boxwing structure. The cabin services and the emergency evacuation procedures are the same of a conventional aircraft. Finally, the freight capability can be increased compared to current airplanes and the time required for ground operations can be reduced, thanks to a single and continuous cargo deck with multiple doors on both front and rear.

The considered PrP baseline configuration, also referred in the following as Milestone 1 configuration (MS1), is illustrated in Fig. 1 and thoroughly described in the companion paper [5]. This configuration features a boxwing and a fuselage with a nearly elliptic cross-section and a total length of $44 \mathrm{~m}$, allowing the accommodation of 310 passengers. Note that this configuration does not include the vertical tails. The total wingspan is of $36 \mathrm{~m}$ with a reference wing surface of $266.7 \mathrm{~m}^{2}$ and a mean aerodynamic chord of $4.31 \mathrm{~m}$. The lower wing planform is classically composed of two trapezoidal parts, with a backward leading-edge sweep angle of $39.78^{\circ}$ for the inner one, and of $40.27^{\circ}$ for the outer one. The dihedral angle varies from $3.81^{\circ}$ to $2.08^{\circ}$ and the taper ratio increases from 0.669 to 0.364 . The trapezoidal upper wing is characterized by a forward leading-edge sweep angle of $21.94^{\circ}$ and a taper ratio of 0.376 , while the dihedral angle is zero. Both the upper and the lower bended wing tips are geometrically described in the $y z$ plane as a circular arc of $90.0^{\circ}$, with a radius of $0.5 \mathrm{~m}$. The vertical wing geometry follows from the connection of the lower and the upper surfaces at their relative positions, and features a backward sweep angle of $51.48^{\circ}$. The same airfoil shape is employed for the whole boxwing; the corresponding reference airfoil geometry is also illustrated in Fig. 1(c).

In addition to the full PrP configuration, the isolated boxwing lifting-system is also considered for aerodynamic analyses and the obtained results compared to those of the PrP. For this purpose, in order to perform CFD calculations on the isolated boxwing, its original geometry has been modified by extrapolating the lower inboard portion of the wing to the symmetry plane $y=0$. Note that in the following the same reference surface is employed in the computation of the aerodynamic coefficients for both the full PrP baseline configuration and the isolated boxwing. As already mentioned, optimization studies have been carried out only on the isolated boxwing liftingsystem.

\section{Methodology}

\section{A. High-Fidelity CFD computations}

High fidelity CFD analyses have been carried out by means of RANS computations using the in-house ONERA cellcentred finite-volume solver elsA (ONERA-Airbus-Safran property). A Chimera approach is employed on purpose with body-fitted structured grids being generated around the boxwing system, the fuselage and the wing-fuselage junction. A Cartesian-octree background grid is also created automatically using the ONERA Cassiopée software library [6], with an extension of $\sim 400 \mathrm{~m}$ away from the aircraft surface along the $x y z$ directions. All these grids are shown in Fig. 2. The normal wall spacing is kept almost uniform everywhere with a size of $\sim 5 \mu \mathrm{m}$, corresponding to a maximum $y^{+} \sim 0.8$ for the considered cruise conditions of $M=0.79$ and altitude of $11.0 \mathrm{~km}$. Once the different grids are assembled, the total number of cells for the half aircraft model employed in the present computations is of $\sim 27.0 \times 10^{6}$. In the case of the isolated boxwing the total mesh size (still referred to the half model) reduces to $\sim 17.0 \times 10^{6}$. For both configurations no-slip boundary conditions are applied on the solid surfaces while on the boundary of the Cartesian background grid, symmetry conditions applies at $y=0$ and far-field conditions are imposed on the remaining boundaries. The RANS equations with the QCR version of the Spalart-Allmaras turbulence model [7-8] and the Jameson scheme [9] for the inviscid flux discretization are converged towards a steady solution by using a backward-Euler pseudo time stepping technique with a LUSSOR implicit stage and a multigrid acceleration. 
A typical convergence history in terms of numerical residuals and drag force is shown in Fig. 3, where the variation of the drag coefficient over the last 2000 iterations is of 0.01 d.c. $(1$ d.c. $=0.0001)$.

a)

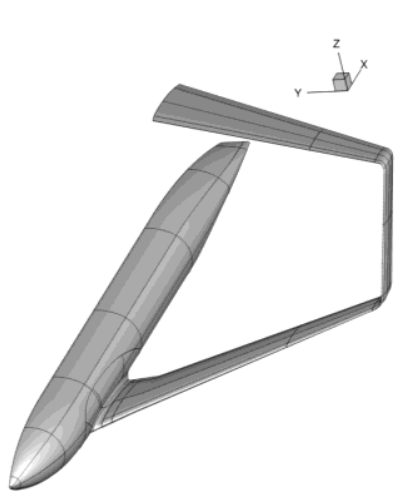

b)
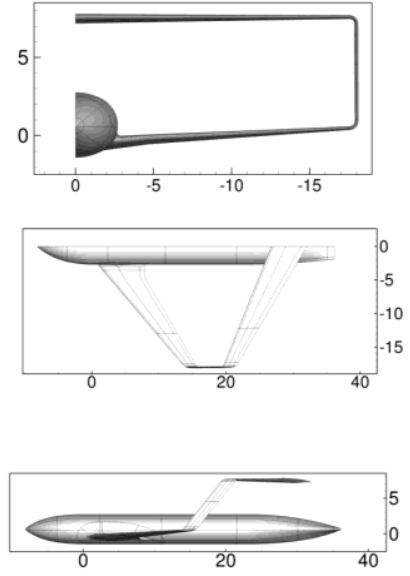

c)

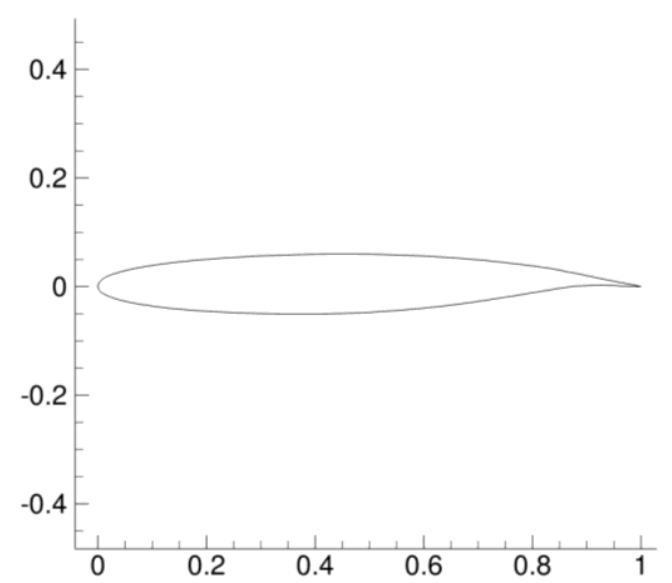

Fig. 1. PrP baseline configuration. (a) Isometric view. (b) Front, top and side views (unit length in m). (e) Reference airfoil shape.

a)

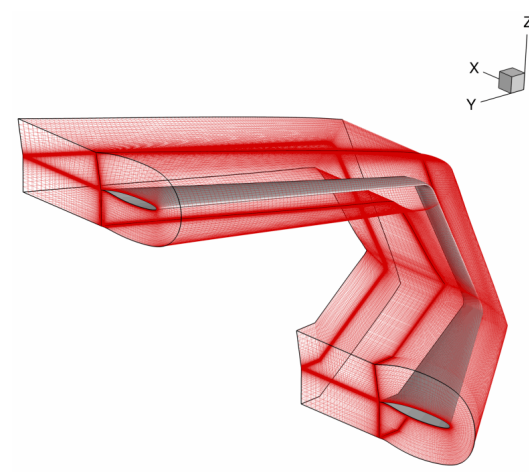

c)

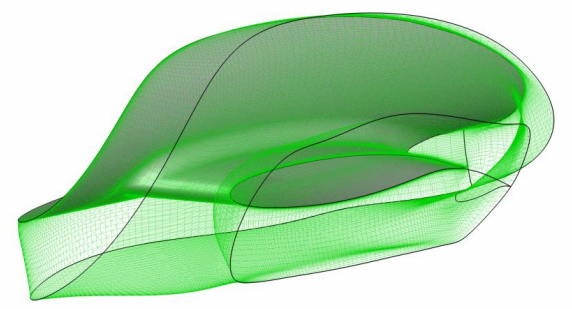

b)

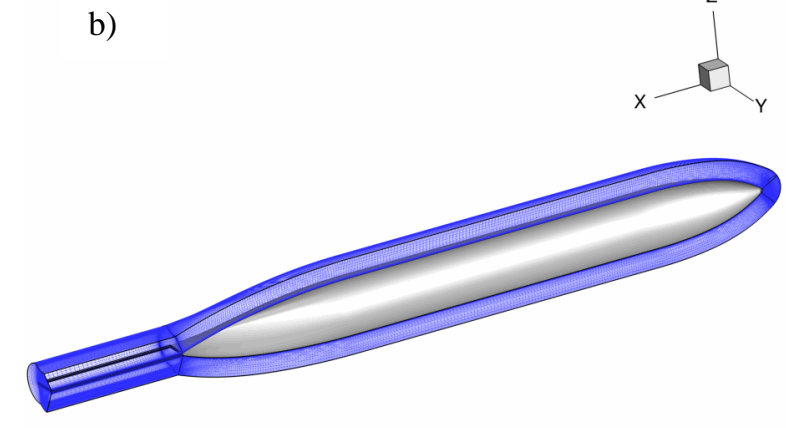

d)

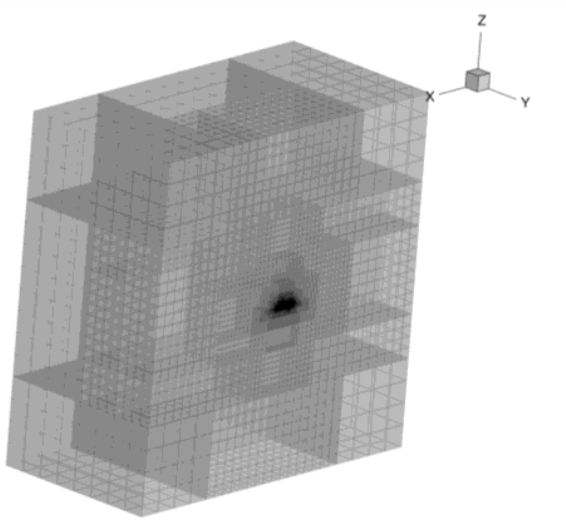

Fig. 2. PrP chimera mesh components. (a) Boxwing mesh with $\sim 15.3 \times 10^{6}$ cells. (b) Fuselage mesh with $\sim 7.8 \times 10^{6}$ cells. (c) Fuselage-wing junction mesh (collar grid) with $\sim 2.7 \times 10^{6}$ cells. (d) Background Cartesian octree mesh with $\sim 1.1 \times 10^{6}$ cells. 


\section{B. Far-field drag post-processing $(f f d)$}

The PrP aerodynamic performances, i.e. its aerodynamic coefficients, have been calculated from the obtained CFD solutions using the far-field drag extraction post-processor ffd developed at ONERA. Indeed this software enables a refined drag evaluation from converged CFD flow fields through an accurate breakdown into lift-induced, viscous (sum of friction and viscous pressure) and wave components. The far-field drag formulation implemented in $f f d$ is derived from that introduced by Van der Vooren and Destarac in Ref. [10]. Further details of the ONERA's so-called one vector variant can be found in Ref. [11]. Without entering into theoretical and computational details we just recall here that an exact near-field balance yields:

$$
C D_{p}=C D_{v p}+C D_{w}+C D_{i}+C D_{s p}
$$

where $C D_{p}$ is the pressure drag, $C D_{v p}$ the viscous pressure drag, $C D_{w}$ the wave drag, $C D_{i}$ the induced drag and $C D_{s p}$ the spurious drag coefficients. The viscous drag is defined by:

$$
C D_{v}=C D_{v p}+C D_{f}
$$

where $C D_{f}$ is the friction drag coefficient. The overall near-field drag is given by:

$$
C D_{n f}=C D_{p}+C D_{f},
$$

and the physical (i.e. without spurious drag) far-field drag is expressed as:

$$
C D_{f f}=C D_{v p}+C D_{w}+C D_{i}+C D_{f} .
$$

a)

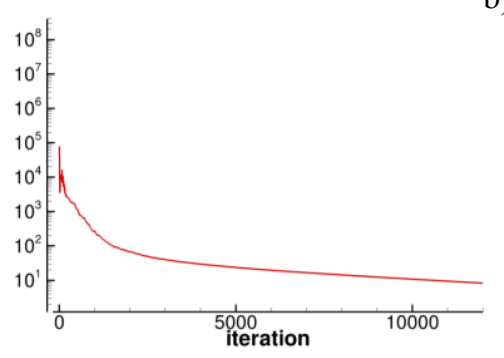

b)

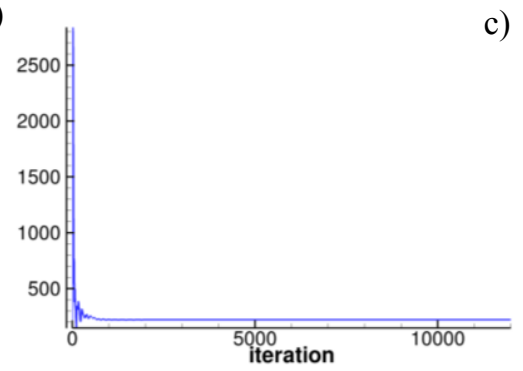

c)

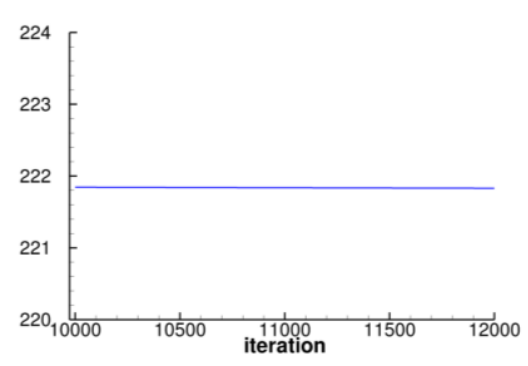

Fig. 3. Convergence history of RANS computations. (a) Mass conservation equation residual. (b) Drag coefficient (d.c.). (c) Enlarged view of the drag coefficient values over the last 2000 iterations. These results are obtained for the PrP baseline configuration at an incidence of $0.0^{\circ}$.

\section{Cruise performance optimization (CANOE)}

In order to improve the aerodynamic performance of the isolated boxwing lifting-system at cruise, aerodynamic optimizations were performed using the CANOE suite developed at ONERA. This module is based on Euler CFD computations performed using the $S U^{2}$ code [12] and on the in-house ONERA far-field drag decomposition tool ffd presented above. As previously mentioned, this tool enables to compute, from a converged CFD flow field, the different physical drag components (viscous, wave and induced) and thus it allows the filtering of the non-physical drag contribution due to numerical dissipation, the so-called spurious drag. Thanks to this approach, the sensitivity of the total drag with respect to the mesh is drastically reduced compared to classical near-field integration methods. In CANOE the aircraft surface mesh is automatically created from its geometrical representation defined through the aircraft modeler OpenVSP [13], while the final unstructured tetrahedral volume mesh (employed for the Euler computations) is generated using tetgen [14]. In order to ensure an accurate and reliable evaluation of the total drag 
during the optimization process, a detailed parametric study with respect to mesh parameters has been carried out and coupled with the post-processing procedure using $f f d$. An example of the results produced by CANOE is shown in Fig. 4 where pressure coefficient distribution and wave drag sources in the flow field are depicted for a generic tube and wing configuration.

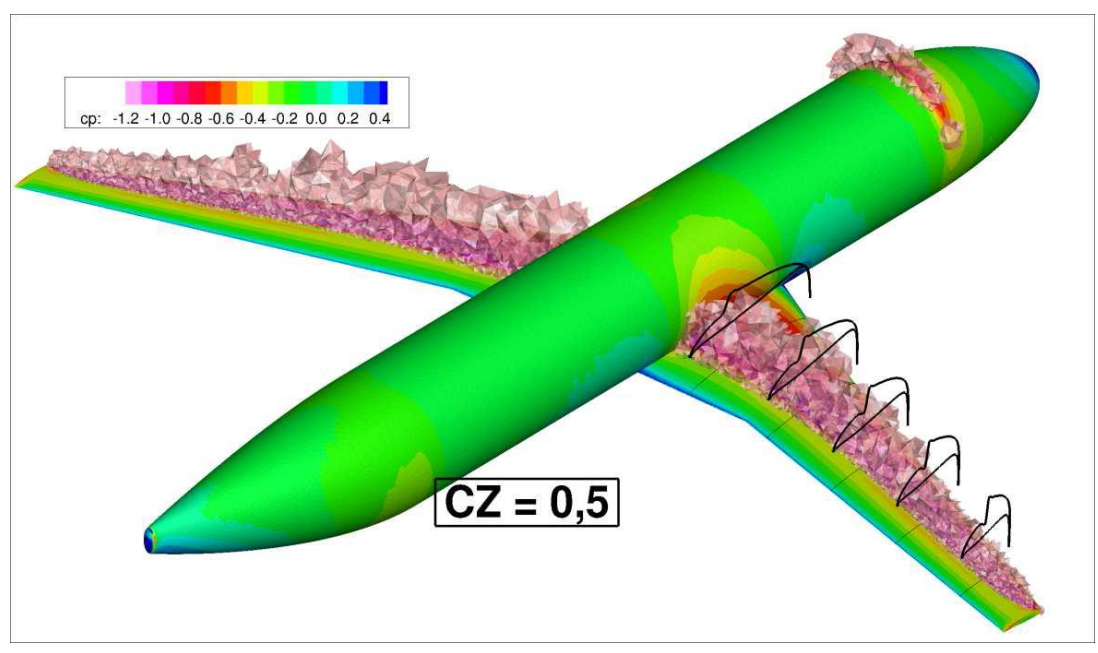

Fig. 4. Euler-based CFD computations performed by $\mathrm{SU}^{2}$ with far-field drag decomposition using ONERA $f f d$ code integrated in the CANOE suite.

\section{Boxwing Geometry Parameterization}

The isolated boxwing lifting system is parameterized by 8 control sections where both twist angle and camber parameters can be selected to define the design space. These sections are illustrated in Fig. 5. It is worthwhile to note that in this case the twist angle is assumed positive according to the boxwing axis orientation from the lower to the upper wing. This convention results in a nose-up twist angle assuming positive and negative values for the lower and upper wings, respectively. In addition the twist is assumed to linearly vary from one control section to the adjacent ones. Two configurations are considered: the original MS1 boxwing, named hereafter "UNIPI" and a modified one by the introduction of new airfoil shapes, specifically designed for transonic conditions, during several ONERA internal research projects, among which, the NOVA project [18]. This new boxwing configuration will be referred in the following as the "ONERA" configuration. In order to understand the impact of these different design parameters and get relevant sensitivity information for the boxwing design, the number of design variables has been progressively increased during the optimization studies.

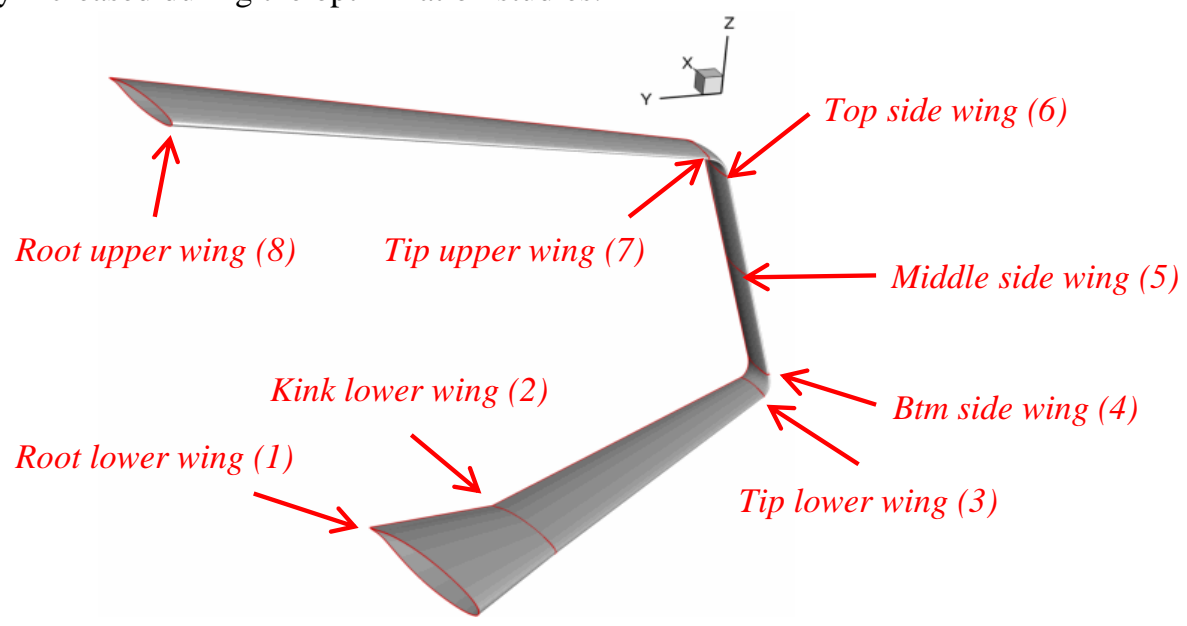

Fig. 5. Control sections for the parameterization of the boxwing geometry. 


\section{Multipoint Constrained Optimization Problem}

In the performed investigations, the optimization problem has been defined in order to improve the aerodynamic performance during the entire cruise phase. For this purpose, a multipoint formulation is adopted with the objective of minimizing the sum of the total drag for 3 constrained lift conditions that are $C L=0.45,0.50,0.55$. The corresponding values of the angle of attack are also introduced as independent variables for the optimization problem. Gradient-based computations, using a finite difference approach, are performed in order to optimize the twist distributions or/and the local airfoil camber for the considered boxwing control sections. The modified (DOTMMFD) method of feasible directions of van der Plaats [15-16] available within the Dakota optimization library $[17]$ is employed to run the optimization process.

\section{Results}

\section{A. Cruise Aerodynamic Performance}

CFD computations of the full PrP configuration and of the isolated boxwing lifting system are performed for the cruise conditions of $M=0.79$ and altitude of $11.0 \mathrm{~km}$, with an $A o A$ varying in the range of [-3.0,3.0 $0^{\circ}$, using a step of $0.25^{\circ}\left(A o A=0.0^{\circ}\right.$ being the cruise design point). The lift $(C L(A o A))$ and polar $(C L(C D))$ curves are illustrated in Fig. 6(a) and Fig. 6(b), respectively. For both the isolated boxwing and the PrP, the stall onset occurs quite early, at an incidence of $\sim 2.5^{\circ}$, which limits the maximum lift coefficient around $\sim 0.6-0.65$. The downward shift of the lift curve of the PrP compared to the isolated boxwing is due to the increase of the lifting surface for this latter geometry while keeping the same reference surface for the aerodynamic coefficient computation. The polar curves in Fig. 6(b) feature a classical parabolic shape and their relative shift along the $C D$ axis can be mainly ascribed to the fuselage drag penalty. In particular, for the reference cruise conditions of $A o A=0.0^{\circ}(C L=0.4322)$, the corresponding increment of the total drag is equal to $\sim 41$ d.c. A more detailed analysis and comparison of the aerodynamic forces is illustrated in Fig. 7, where the polar curves of Fig. 6(b) are decomposed according to the far-field drag breakdown. The same overall behavior is observed for corresponding drag contributions when comparing the results for the PrP and the isolated boxwing. As expected, the contribution of the viscous dissipation is almost insensitive to the load condition and the shift of $\sim 33$ d.c. between the two curves is clearly due to the increase of the whole body wetted surface by the fuselage. The wave drag is very small at negative angles of attack and gradually increases up to $\sim 50$ d.c. for $C L>0.6$. A sudden drag rise occurs for the viscous pressure drag when $C L$ increases over $\sim 0.55$, thus suggesting the onset of a large scale separation but the wave drag rise is not as regular as expected. This is due to the fact that at low $A o A$, the main wave drag sources are located on the suction side of the lower wing tip (see Fig. 9) whereas at high $A o A$, a separation occurs in this region, which prevents the development of the strong shock described before. As for the induced drag, a nearly quadratic behavior is found with no substantial difference between the PrP and the isolated boxwing. These findings further support the restriction of the optimization process to this last configuration, thus allowing the reduction of the computational cost while providing relevant results for the improvement of the full PrP configuration. For the reference cruise conditions, the induced drag component represents the $\sim 43 \%$ of the total drag. The values of the different drag components for $A o A=0.0^{\circ}$ and $A o A=2.0^{\circ}$ (just before the stall onset) are listed in Table 1 . The aerodynamic efficiency $L / D$ and the equivalent Oswald efficiency $e$ are illustrated in Fig. 8 as a function of $C L$. Not surprisingly, the aerodynamic efficiency is always higher for the isolated boxwing. For both cases the maximum efficiency is achieved close to the cruise design point, around $C L \sim 0.4$ with a value of $\sim 24.1$ for the isolated lifting system and of $\sim 19.4$ for the full PrP configuration. As expected, for a boxwing architecture, the Oswald efficiency is always greater than 1 , with $\mathrm{e}=1.28$ for the $\operatorname{PrP}$ at $A o A=0.0^{\circ}$. A small reduction of $e$ is also observed w.r.t. the isolated boxwing. In addition, it is worthwhile to note that the maximum value of $e$ is not achieved at the maximum aerodynamic efficiency conditions but at $C L \sim 0.55$. Then an abrupt decrease of $e$ occurs at increased lift conditions. However it should be noted that this incipient stall condition fall outside the limits of the Prandtl lifting line model and the related definition of $e$ is rather pointless. 
a)

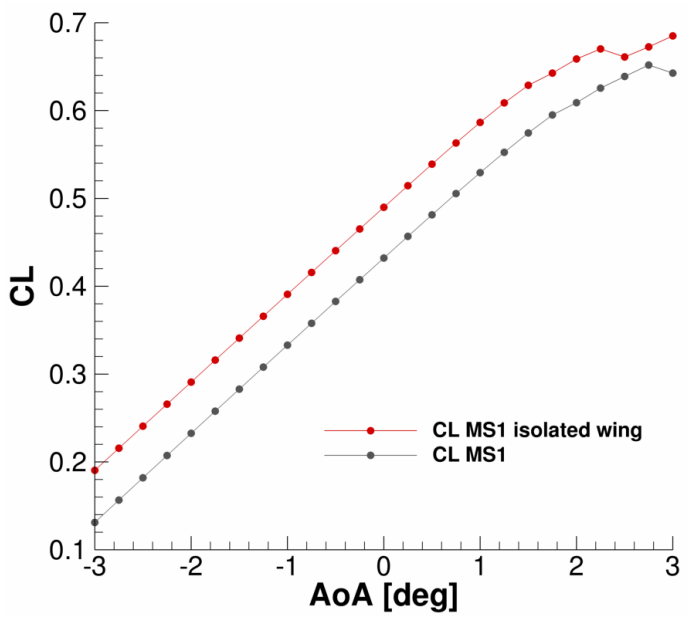

b)

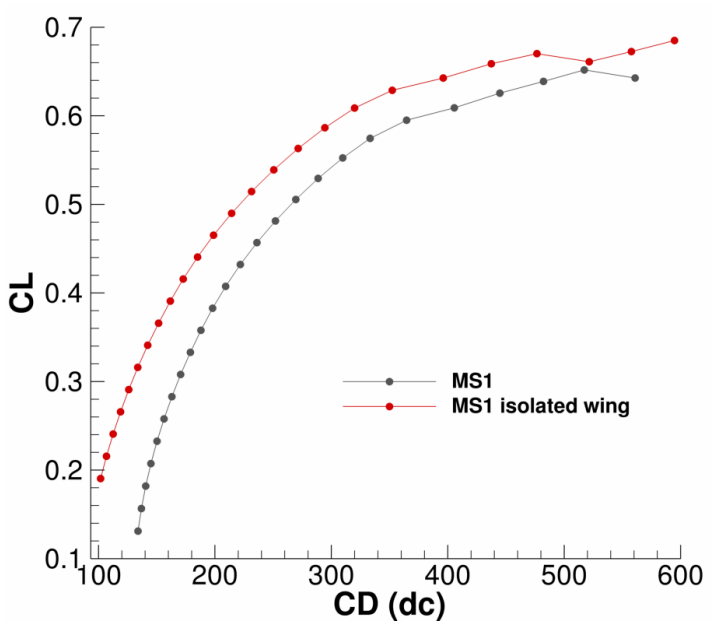

Fig. 6. Aerodynamic coefficients computed for the PrP baseline configuration (MS1) and the corresponding isolated boxwing: each dot corresponds to a CFD run at a given AoA. (a) Lift curve. (b) Polar curve.

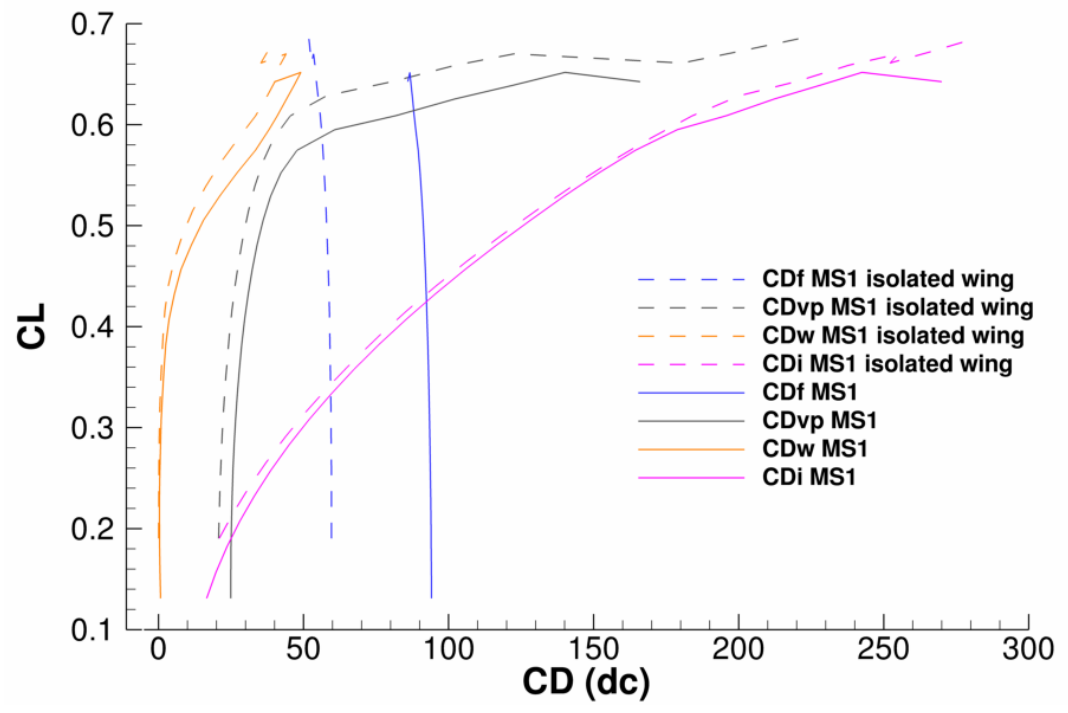

Fig. 7. PrP and isolated boxwing polar curve breakdown according to the far-field drag decomposition postprocessing. 


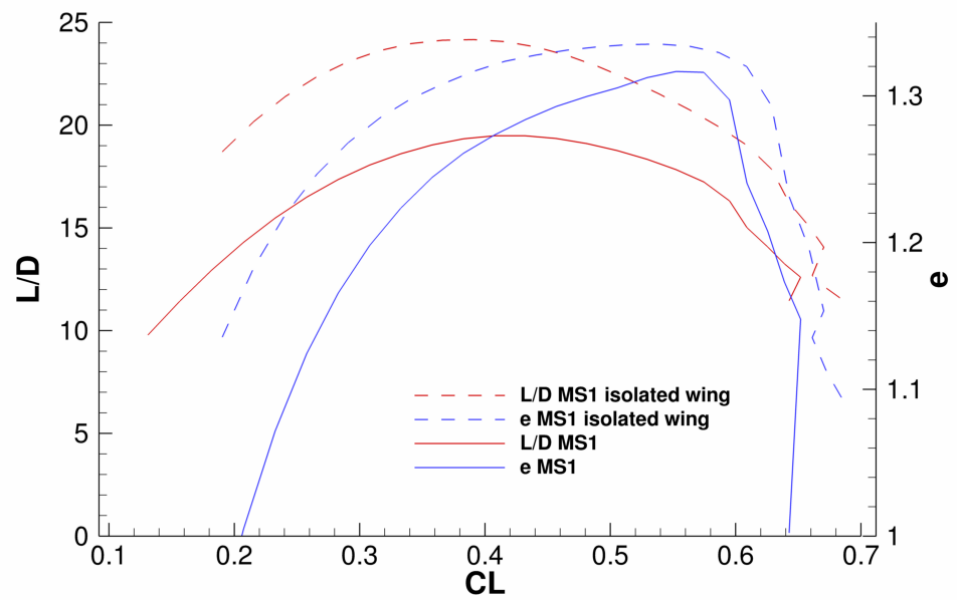

Fig. 8. Aerodynamic efficiency and equivalent Oswald factor as a function of the lift coefficient for both the full PrP configuration and the isolated boxwing.

\begin{tabular}{ccccccccc}
\hline $\boldsymbol{A o A}^{\circ}$ & $\boldsymbol{C L}$ & $\boldsymbol{C D}$ & $\boldsymbol{C D} \boldsymbol{f}$ & $\boldsymbol{C D} \boldsymbol{v} \boldsymbol{p}$ & $\boldsymbol{C D w}$ & $\boldsymbol{C D i}$ & $\boldsymbol{L} / \boldsymbol{D}$ & $\boldsymbol{e}$ \\
\hline $\mathbf{0 . 0}^{\circ}$ & 0.432 & 221.83 & 92.29 & 30.90 & 5.46 & 95.31 & 19.4 & 1.28 \\
$\mathbf{2 . 0}^{\circ}$ & 0.609 & 405.63 & 88.13 & 81.89 & 40.96 & 195.84 & 15.0 & 1.24 \\
\hline
\end{tabular}

Table 1. Lift and drag coefficients of the PrP baseline configuration with far-field drag breakdown for cruise conditions $A o A=0.0^{\circ}$ and before the stall at $A o A=2.0^{\circ}$.

\section{B. $C p$ distributions}

The computed pressure coefficient $C p$ for $A o A=0.0^{\circ}, A o A=0.75^{\circ}$ and $A o A=2.0^{\circ}$ is depicted in Fig. 9. Note that in this figure only the results concerning the PrP are presented. At cruise conditions, $A o A=0.0^{\circ}$, Fig. $9(\mathrm{a}, \mathrm{d})$, a strong shock-wave is observed at the tip of the lower wing. As the incidence is increased the shock becomes more intense, Fig. 9(e), and then for $A o A=2.0^{\circ}(C L=0.6090)$ it disappears, due to the induced flow separation. At the same time a lambda-shock structure develops on the inboard portion of the lower wing, as shown in Fig. 9(f).

\section{Boxwing optimization results}

\section{Euler-based optimization studies}

Various optimization studies have been performed for both the considered configurations, i.e. UNIPI and ONERA, in order to investigate the effect of the different design variables on the boxwing aerodynamic performance in terms of maximum achievable gain from optimized designs. More precisely we carried out optimization studies based on:

- Twist optimization;

- Camber optimization;

- The simultaneous optimization of both twist and camber design variables;

Moreover, for the optimization study combining together all the considered airfoil section parameters, different strategies were adopted and tested to define the initial guess, i.e. starting the optimization process either from the baseline design or from the optimal solution previously obtained by considering each set of parameters alone. This strategy allowed us to partially handle the existence of local minima in the design space, given the local nature of the adopted optimization algorithm. 
a)

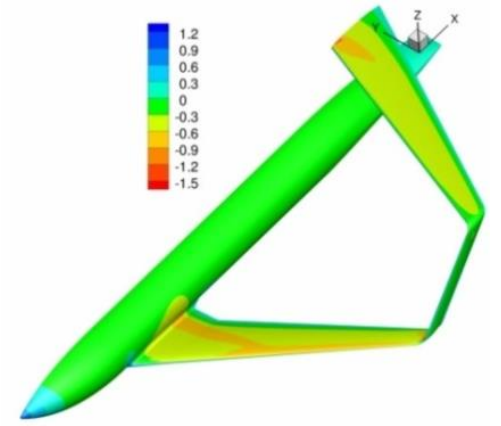

d)

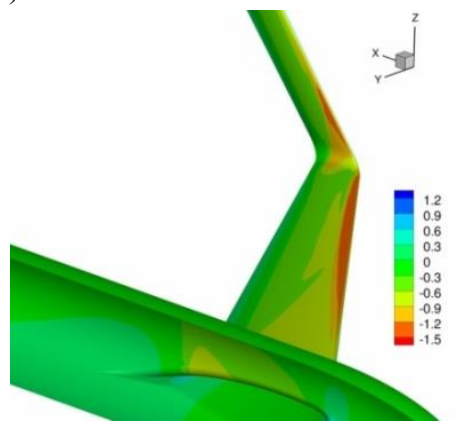

b)

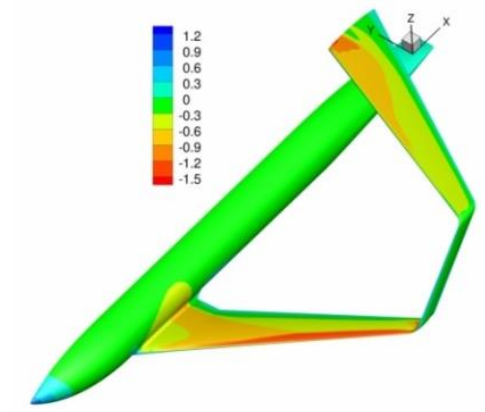

e)

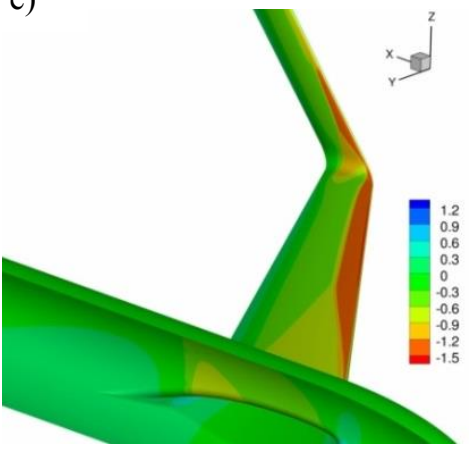

c)
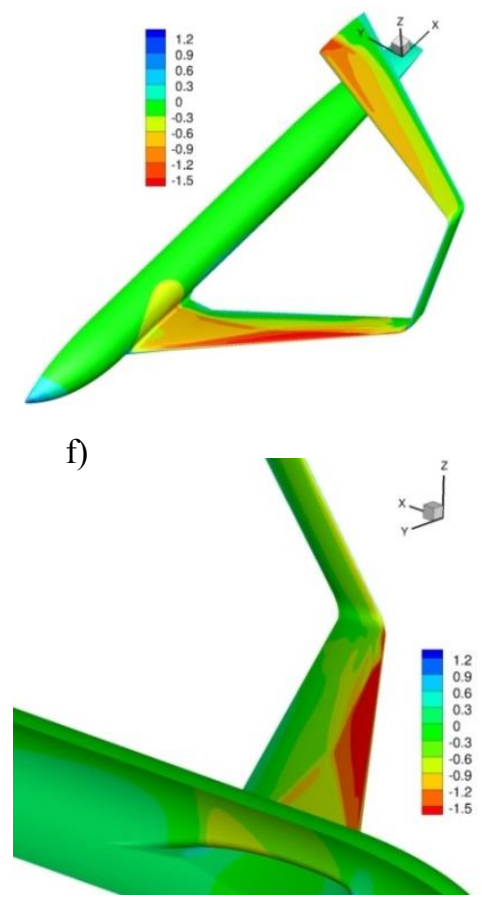

Fig. 9. PrP $C p$ distribution at different angles of attack. (a,d) $A o A=0^{\circ}, C L=0.4322$. $(\mathrm{b}, \mathrm{e}) A o A=0.75^{\circ}$, $C L=0.5056$. $(\mathrm{c}, \mathrm{f}) \mathrm{AoA}=2.0^{\circ}, C L=0.6090$.

All the performed optimization studies have achieved a reduction of the total drag coefficient for the three design points, while satisfying the corresponding lift constraints. The best performance is obtained by including both twist and camber variables in the optimization process, with UNIPI design performing better than the ONERA one in terms of total Euler drag, as illustrated in Table 2.

\begin{tabular}{ccccc} 
& $\begin{array}{c}C D_{w} \\
(\mathbf{d . c})\end{array}$ & $\begin{array}{c}C D_{i} \\
(\mathbf{d . c} .)\end{array}$ & $\begin{array}{c}C D_{i}+C D_{w} \\
(\mathbf{d . c} .)\end{array}$ & $\boldsymbol{e}$ \\
\hline UNIPI Initial & 8.30 & 96.51 & 104.81 & 1.362 \\
& 14.96 & 120.27 & 135.23 & 1.371 \\
& 26.30 & 146.97 & 173.27 & 1.375 \\
ONERA Initial & 10.4 & 84.7 & 95.1 & 1.401 \\
& 18.4 & 106.7 & 125.1 & 1.402 \\
UNIPI Optimal & 31.2 & 131.4 & 162.6 & 1.399 \\
& 3.77 & 92.58 & 96.35 & 1.431 \\
ONERA Optimal & 6.27 & 113.81 & 120.08 & 1.437 \\
& 11.29 & 137.81 & 149.1 & 1.436 \\
& 1.5 & 98.1 & 99.6 & 1.349 \\
& 2.6 & 119.9 & 122.5 & 1.363 \\
& 5.1 & 144.2 & 149.3 & 1.372 \\
\hline
\end{tabular}

Table 2. Comparison of wave drag $\left(C D_{w}\right)$, induced drag $\left(C D_{i}\right)$, total Euler drag $(C D w+C D i)$ and Oswald efficiency $(e)$ for both initial and optimized UNIPI and ONERA configurations according to Euler computations. Note that the optimized configurations refer to the optimization study performed by considering both twist and camber design variables. 


\begin{tabular}{cccc} 
Design Variable & Starting Value & UNIPI Optimal & ONERA Optimal \\
\hline Twist section 1 & 2.72 & 2.42 & 3.34 \\
Twist section 2 & 3.87 & 3.49 & 2.98 \\
Twist section 3 & 1.47 & 1.02 & -2.20 \\
Twist section 4 & 1.44 & 1.28 & 1.49 \\
Twist section 5 & 0.04 & -0.23 & 0.24 \\
Twist section 6 & -1.36 & -1.47 & -0.59 \\
Twist section 7 & -1.37 & -1.84 & -2.10 \\
Twist section 8 & -3.67 & -3.82 & -2.82 \\
\hline
\end{tabular}

Table 3. Initial and optimized values of the twist angles (degree) for UNIPI and ONERA boxwing configurations. The optimal values refer to the optimization study performed by also including camber design parameters.

a)

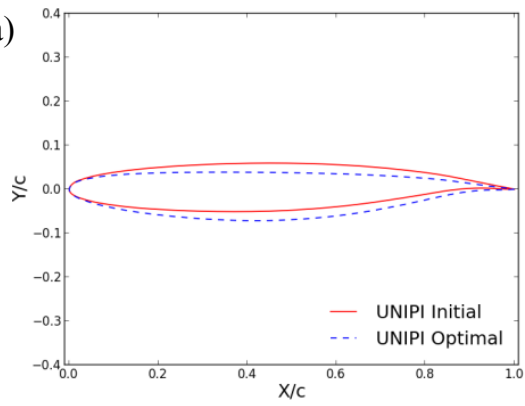

d)

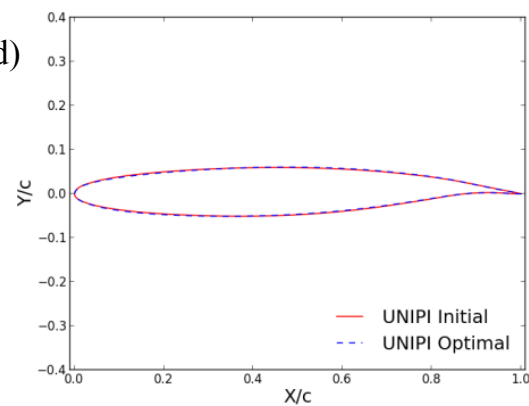

g)

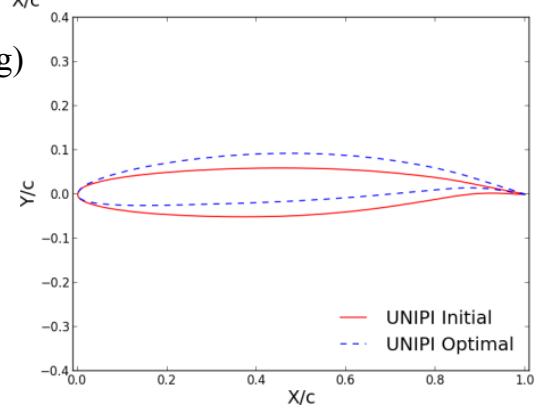

b)

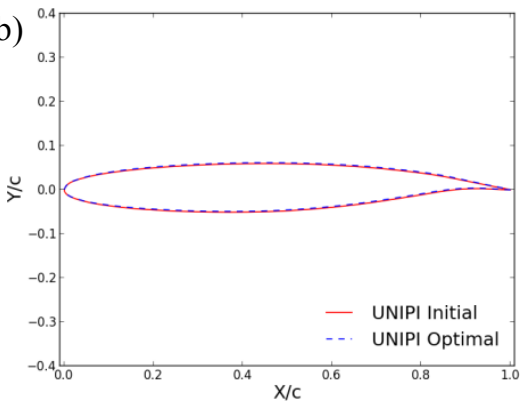

e)

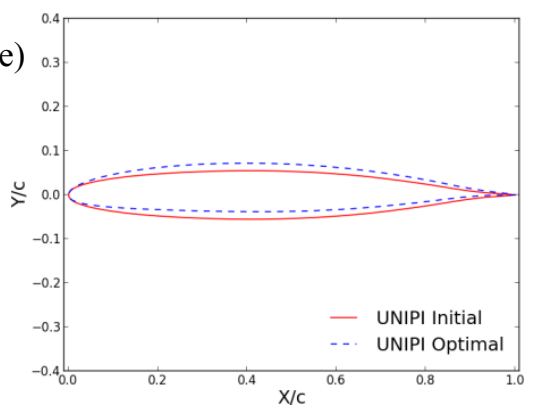

h)

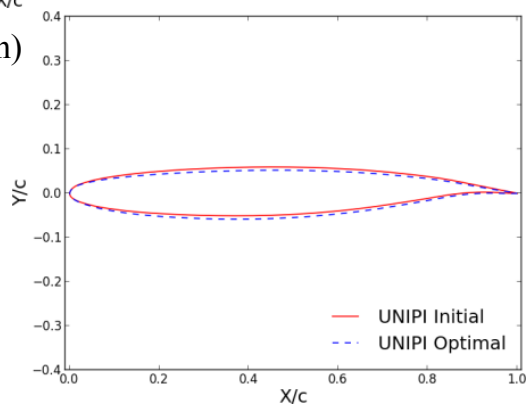

c)
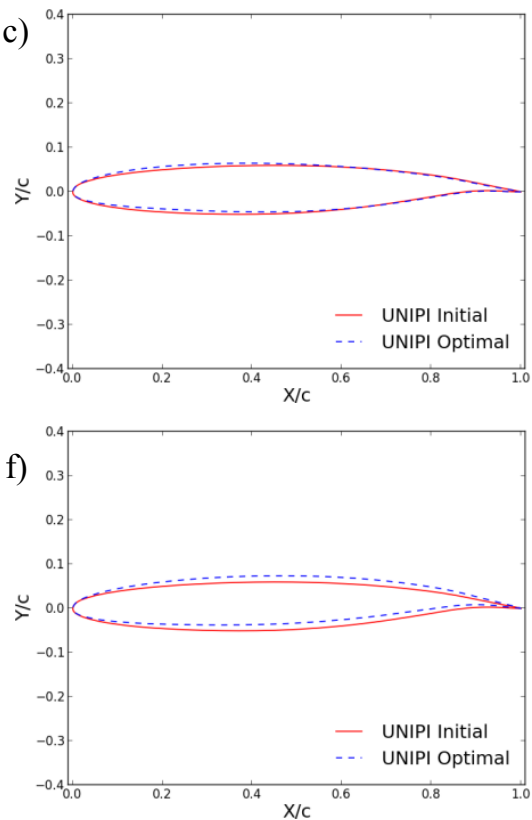

Fig. 10: Comparison of initial and optimized airfoils shapes (camber effect only) for the UNIPI boxwing configuration: (a) section 1; (b) section 2; (c) section 3; (d) section 4; (e) section 5; (f) section 6; (g) section 7; (h) section 8. Note that these optimized shapes refer to the optimization study performed by including both twist and camber design variables. 
For both UNIPI and ONERA optimized configurations (using twist and camber variables), the corresponding optimal values of twist angle are reported in Table 3. In addition, for each boxwing section, a comparison between the original and the optimized airfoil shapes is illustrated in Fig. 10 for the UNIPI configuration only, while keeping ONERA airfoil shapes and their modifications confidential. It is worthwhile to observe that the most important modifications occur at section 7 (Fig. 10g), i.e. at the tip section of the upper wing where the airfoil camber results appreciably increased. A similar effect can also be observed at section 6 (Fig. 10f), while at section 1 (Fig. 10a), the camber is reduced. Similar considerations hold for the ONERA design, except for section 7, where the UNIPI airfoil shape is quite different from the corresponding ONERA one, due to the introduction of an airfoil specifically designed for forward swept wings. A more detailed comparison in terms of wave drag, induced drag and Oswald efficiency (e), among the baseline and the optimized boxwing designs is also presented in Table 2. For both UNIPI and ONERA configurations, the optimization process successfully achieved an important improvement of the overall aerodynamic performance at each design point, up to 24 d.c. for the UNIPI configuration at $C L=0.55$. It is interesting to observe that for the two configurations, the optimization process lead to a different compromise in terms of wave and induced drag. The optimal UNIPI design achieves the best performance in terms of induced drag with very high values of Oswald efficiency, $e \sim 1.43$. However the reduction of the $C D_{w}$ component is not as relevant as for the ONERA configuration, which features less than half of the wave drag of the UNIPI one at each target lift condition, but also a lower Oswald efficiency, $e=1.35-1.37$. Based on these results it is likely that the ONERA configuration can provide better performance at higher lift coefficients as well as in terms of drag-divergence Mach number.

\section{RANS - FFD assessment and analysis of the optimized designs}

The aerodynamic performance of both UNIPI and ONERA optimized configurations are here investigated based on high-fidelity RANS computations and their far-field drag post-processing. The comparison with the corresponding baseline designs is firstly presented in Fig. 11 in terms of classical lift and drag polar curves. All the considered designs feature almost the same slope of the lift coefficient curve, Fig. 11(a), as expected according to the common wing-planform geometry. The lift curves essentially differ for the value of the zero-lift angle of attack. With reference to the polar curve, Fig. 11(b), for both UNIPI and ONERA configurations, the optimization process successfully lead to ameliorate the aerodynamic performance for $C L>0.4$, with a considerable improvement at higher values of the lift-coefficient. In order to better understand this behavior, the corresponding far-field drag breakdown is inspected. In Table 4 a detailed comparison of the different drag contributions from Euler and RANS computations for a fixed lift coefficient value of $C L=0.5$ is reported. Consistently with past studies in the literature, Euler computations tend to overestimate and underestimate the wave drag and the induced drag components, respectively. An extensive RANS-based comparison in terms of polar curves among the different designs is illustrated in Fig. 12. As expected, the variation of the friction drag is almost negligible. Conversely, the achieved performance improvement for $C L>0.4$ can be essentially ascribed to the important reduction of the wave drag and to the associated impact in terms of viscous-pressure drag, with the delay of shock-induced separation phenomena.

\begin{tabular}{llccccccccc}
\hline Design & Model & $\boldsymbol{A o A}$ & $\boldsymbol{C L}$ & $\boldsymbol{C D}$ & $\boldsymbol{C D}$ & $\boldsymbol{C D}$ & $\boldsymbol{C D}_{w}$ & $\boldsymbol{C D}$ & $\boldsymbol{L} / \boldsymbol{D}$ & $\boldsymbol{e}$ \\
\hline UNIPI & Euler & -0.515 & 0.500 & 206.8 & 58.2 & 28.5 & 6.27 & 113.81 & 24.18 & 1.439 \\
Optimized & RANS & 0.605 & 0.500 & 216.7 & 57.6 & 35.6 & 4.66 & 118.86 & 23.07 & 1.378 \\
ONERA & Euler & -0.518 & 0.500 & 209.2 & 58.2 & 28.5 & 2.6 & 119,9 & 23.90 & 1.366 \\
Optimized & RANS & 0.318 & 0.500 & 210.1 & 59.2 & 25.1 & 0.7 & 125,1 & 23.80 & 1.309 \\
\hline
\end{tabular}

Table 4. Comparison between Euler and RANS based prediction of the aerodynamic performance at $C L=0.5$ for both UNIPI and ONERA optimized designs obtained by considering both twist and camber parameters. Note that all the drag coefficients are reported in terms of drag counts while the incidence, $A o A$, is expressed in degree. 
a)

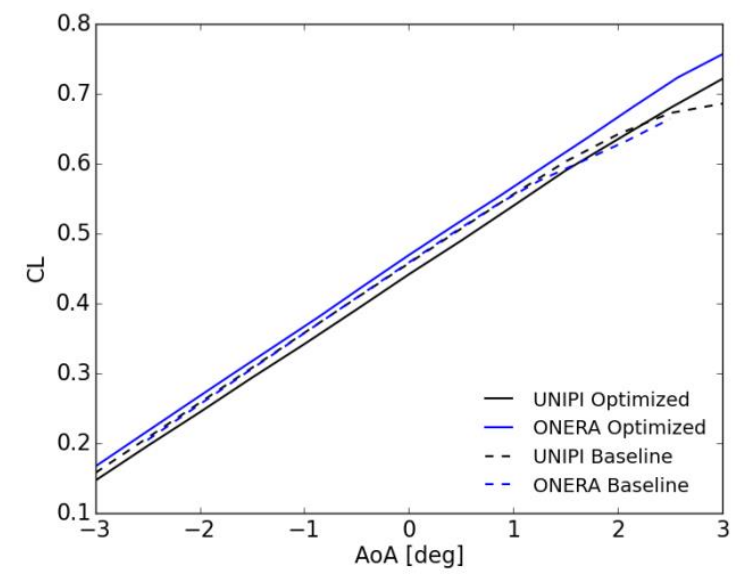

b)

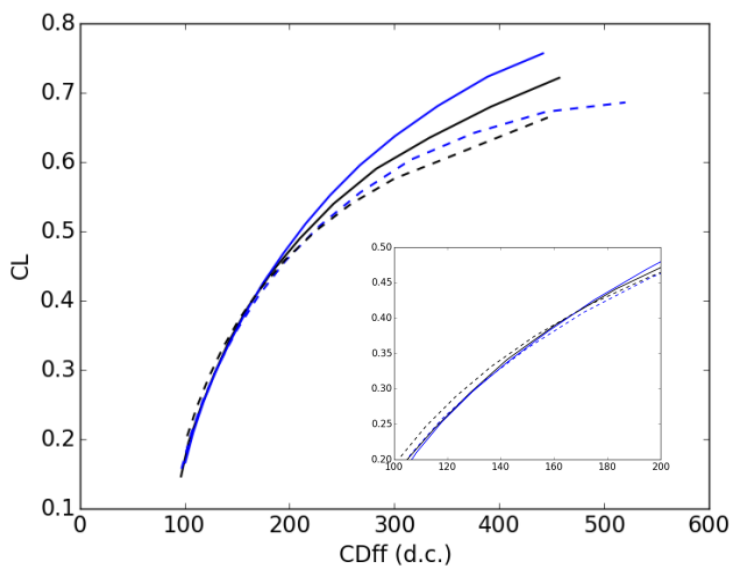

Fig. 11. RANS assessment of UNIPI and ONERA Euler optimized configurations w.r.t. to corresponding baselines. (a) Lift curve. (b) Far-field drag polar curve.

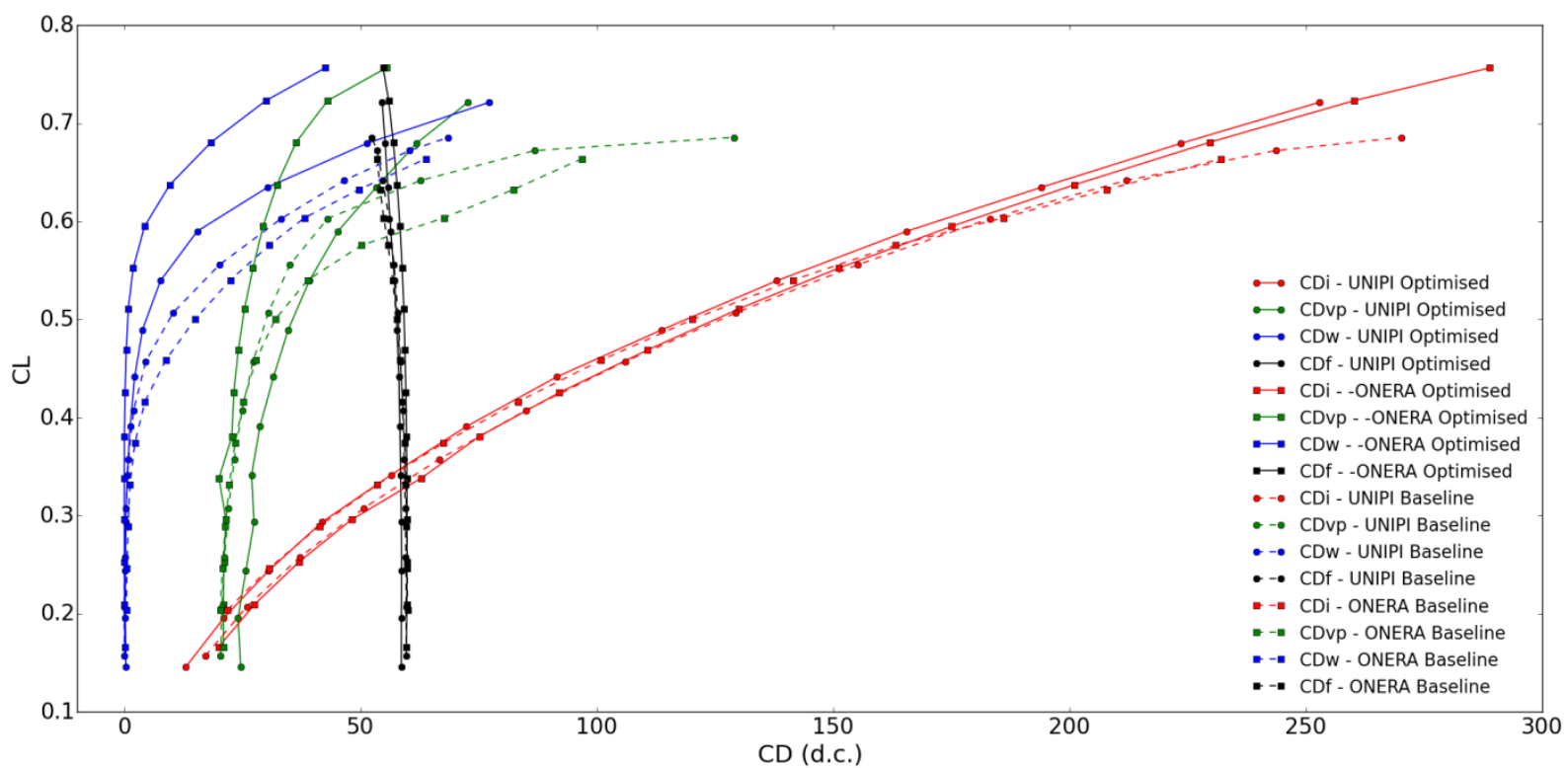

Fig. 12. RANS assessment of UNIPI and ONERA Euler optimized configurations w.r.t. to corresponding baselines: far-field drag breakdown. Optimised configuration refer to the simultaneous optimisation of both twist and camber design variables. 
a)

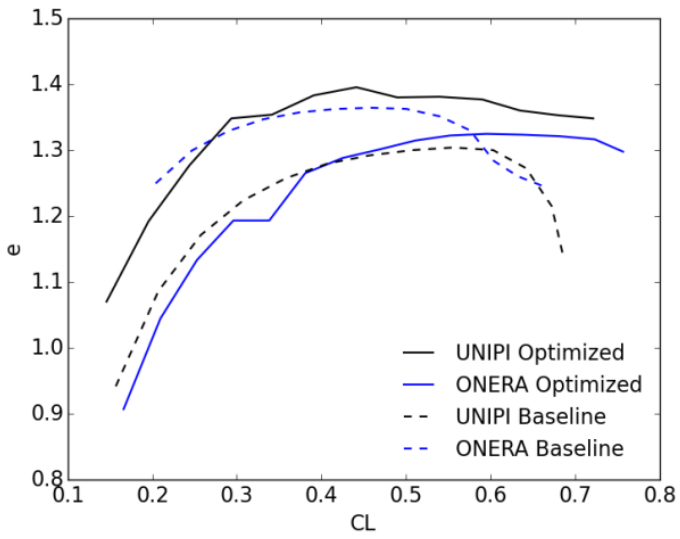

b)

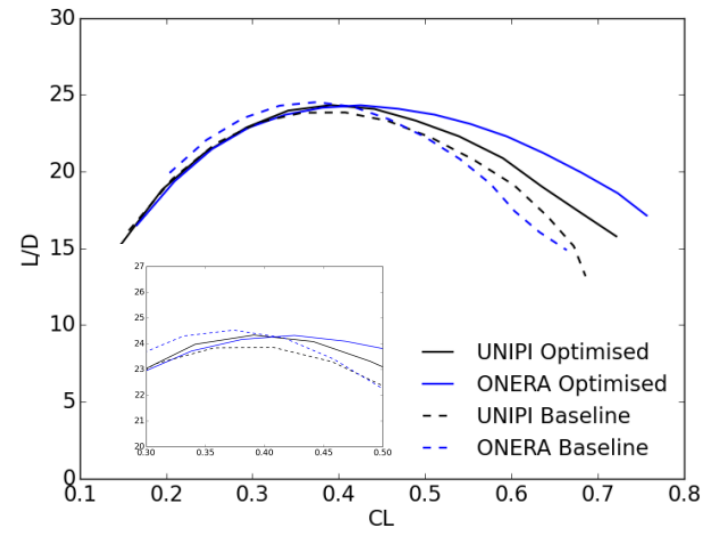

Fig. 13. RANS assessment of UNIPI and ONERA Euler optimized configurations w.r.t. to corresponding baselines: (a) Oswald efficiency. (b) Aerodynamic efficiency. Optimised configuration refer to the simultaneous optimisation of both twist and camber design variables.

a)

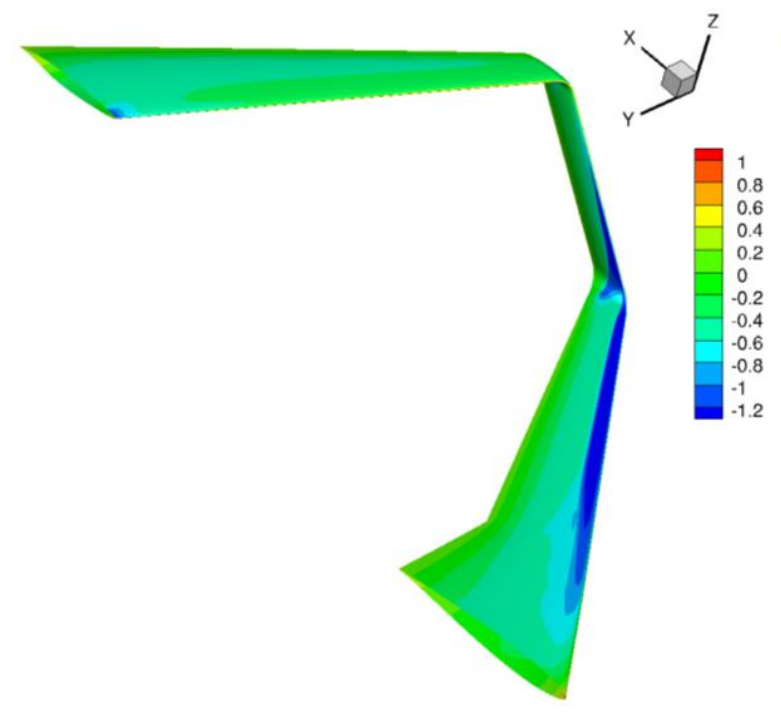

b)

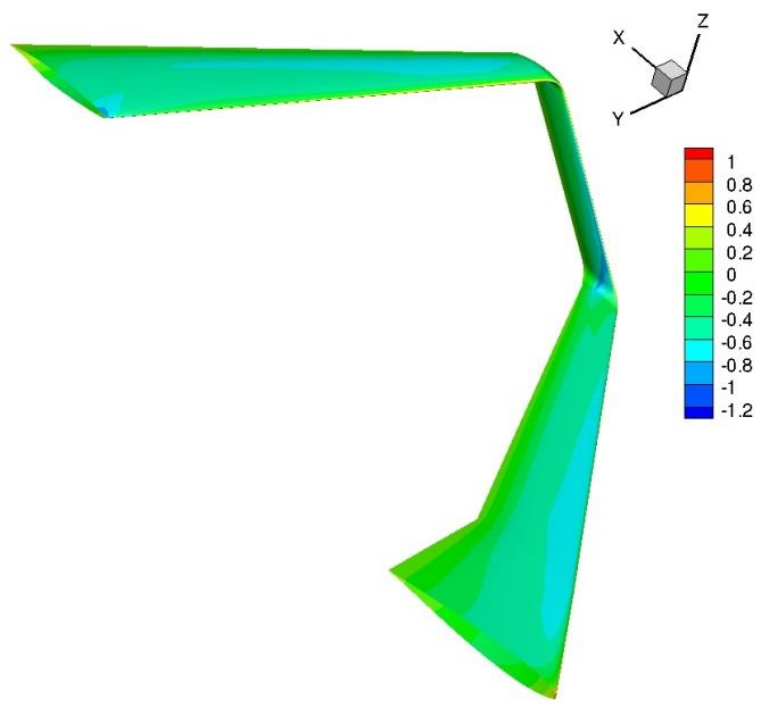

Fig. 14. RANS assessment of ONERA Euler optimized configuration w.r.t. to corresponding baseline: comparison of pressure coefficient $(C p)$ distributions. (a) Baseline at $C L=0.458$. (b) Optimized at $C L=0.469$. Optimised configuration refer to the simultaneous optimisation of both twist and camber design variables. 
a)

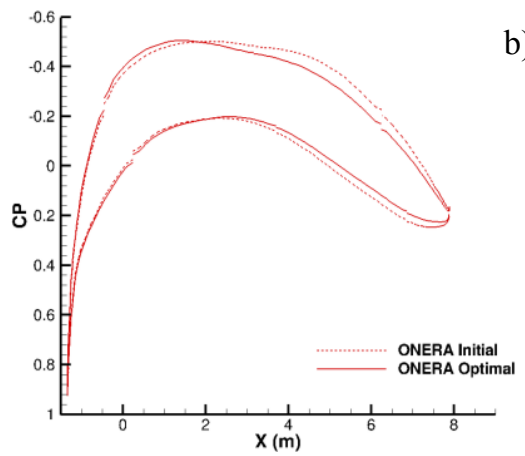

d)

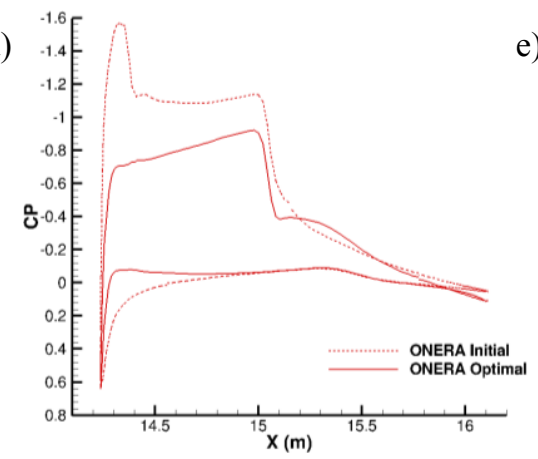

b)

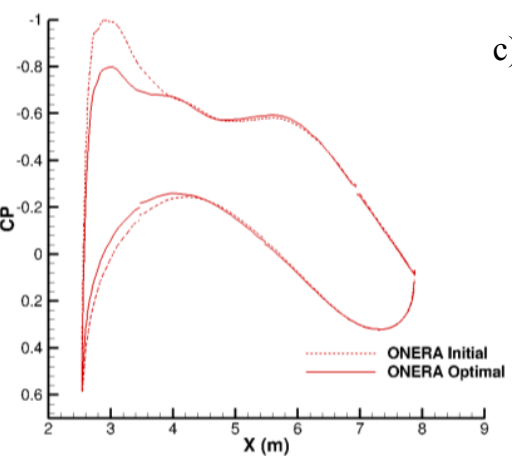

e)

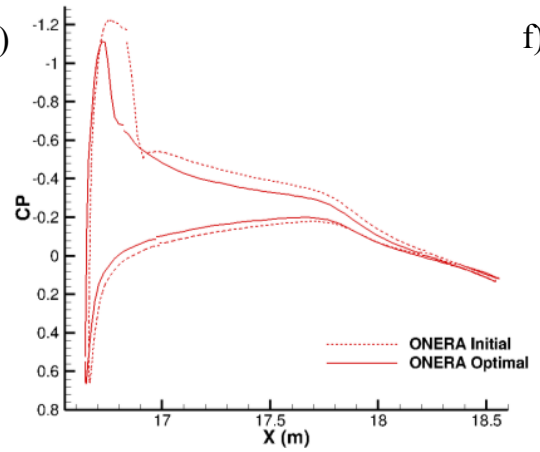

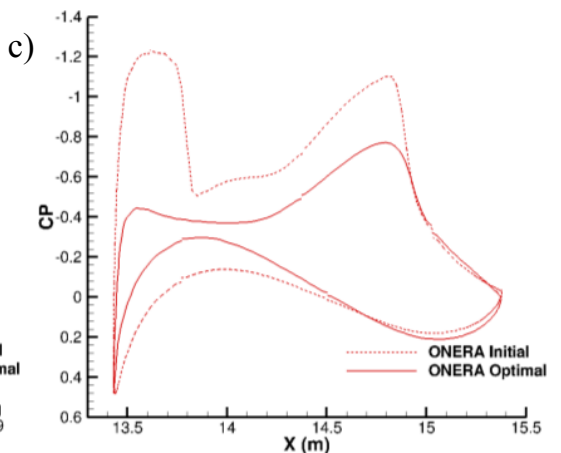

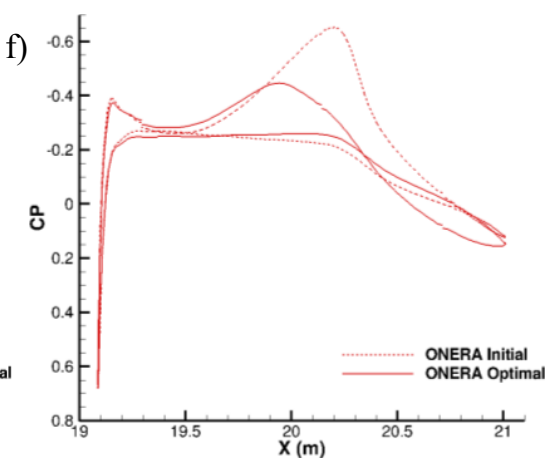

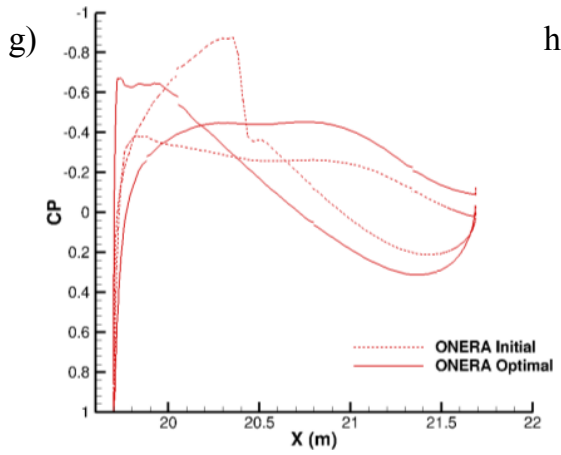

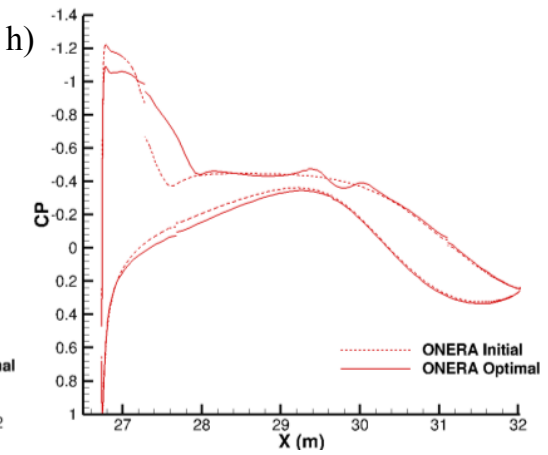

Fig. 15. RANS assessment of ONERA optimized configuration w.r.t. to corresponding baseline: comparison of pressure coefficient $(C p)$ distributions at selected sections: (a) section 1; (b) section 2; (c) section 3; (d) section 4; (e) section 5; (f) section 6; (g) section 7; (h) section 8.

For both these two drag components, the optimized ONERA configuration shows superior performance w.r.t. the optimized UNIPI one, thanks to newly introduced supercritical airfoil shapes. The same is not true for the induced drag component, with the optimized UNIPI configuration achieving higher values of Oswald efficiency as illustrated in Fig. 13(a), thus confirming the trend already observed based on Euler computations. Comparison in terms of total aerodynamic efficiency is then reported in Fig. 13(b) assessing the superior improvement achieved by the ONERA optimized design for $C L>0.4$.

With focus on the ONERA configuration, a detailed comparison of the pressure coefficient distribution between the baseline and the optimized shapes is illustrated in Fig. 14 as well as in Fig. 15 (for the considered reference sections). These results clearly highlight the strong mitigation of the compressibility effects resulting from the optimization process, especially at sections 3 and 4, as well as at sections 6 and 7, where relevant camber modifications have been introduced for the ONERA configuration. 


\section{Conclusions}

A high-fidelity CFD assessment of the transonic performance of the PARSIFAL PrP baseline (MSl) configuration has been presented in this paper with a detailed analysis of the impact of the different drag contributions, according to a far-field drag analysis. The obtained results confirm the expected benefits offered by the boxwing architecture in terms of induced drag in transonic cruise flight conditions $(M=0.79$ and altitude of $11.0 \mathrm{~km})$ with a maximum value of Oswald efficiency of $e \sim 1.32$ for $C L \sim 0.55$. In addition, and with reference to the given aspect ratio value of 4.86, a quite high aerodynamic efficiency is observed with $\mathrm{L} / \mathrm{D}=19.3$. Notwithstanding, the inspection of the skin pressure distributions has also highlighted the occurrence of strong compressibility effects on the PrP forward wing, leading to the onset of a large flow separation at relatively small incidence, thus simultaneously reducing the maximum achievable lift while producing a strong drag rise.

The improvement of the aerodynamic design has then been tackled by focusing the attention on the isolated boxwing configuration with the combined multi-point optimization of the airfoil local twist and camber design parameters, using fast Euler computations and far-field drag extraction. In addition to the reference baseline (UNIPI), a new one is introduced by the adoption of different supercritical airfoil shapes, previously designed at ONERA. For both configurations, the optimizer succeeds in considerably delaying the wave-drag rise and the associated separation phenomena, which is confirmed through RANS computations and far-field drag analysis over both the two optimized designs. It is also interesting to note that the two optimized configurations feature a different trade-off in terms of wave and induced drag, with a small reduction of the Oswald efficiency for the ONERA configuration in favor of a larger shock reduction compared to the optimized UNIPI design, for which Oswald efficiency is instead improved up to $e \sim 1.38$. The ONERA design achieves the best performance in terms aerodynamic efficiency in the range of $C L>0.4$. The reduction of compressibility effects is furthermore confirmed by the detailed inspection of the pressure coefficient distribution: shock waves are still affecting the boxwing architecture at the tip of the lower wing, deserving further and specific design actions based on an ad-hoc local shape parameterization. A first attempt to tackle this aspect and successfully reduce compressibility effects by a tailored design of this region is presented in the companion paper [5] along with additional improvements of the baseline configuration recently achieved.

\section{Acknowledgments}

The present paper concerns part of the activities carried out within the research project PARSIFAL ("Prandtlplane ARchitecture for the Sustainable Improvement of Future AirpLanes"), which has been funded by the European Union under the Horizon 2020 Research and Innovation Program (Grant Agreement n.723149).

\section{References}

[1] Frediani A., Cipolla V., Binante V., Abu Salem K., Maganzi M., "PARSIFAL PROJECT: A BREAKTHROUGH INNOVATION IN AIR TRANSPORT", Proceedings of the XXIV AIDAA International Conference, 18-22 September 2017, Palermo \& Enna (Italy).

[2] Prandtl L., "Induced drag of multiplanes", NACA Technical Note, no. 182, 1924.

[3] Frediani A., “The Prandtl Wing”, Lecture Series Innovative Configurations and Advanced Concepts for Future Civil Aircraft, Von Karman Institute, ISBN 2-930389-62-1, VKI 2005.

[4] Frediani A. and Montanari G., "Best wing system: An exact solution of the Prandtl's problem”. In G. Buttazzo and A. Frediani Ed., Variational Analysis and Aerospace Engineering. Page. 181-211.Springer, 2009.

[5] Cipolla V., Abu Salem K., Picchi Scardaoni M. and Binante V., "Preliminary Design and performance analysis of a box-wing transport aircraft", AIAA SciTech Forum, Orlando, Florida, 2020 (to be published)

[6] http://elsa.onera.fr/Cassiopee/

[7] Spalart, P. R., and Allmaras, S. R., “A One-Equation Model for Aerodynamic Flows”, AIAA paper 92-0439, 30th AIAA

Aerospace Sciences Meeting and Exhibit, Reno, NV, Jan. 6-9, 1992.

[8] Spalart, P. R.,"Strategies for Turbulence Modelling and Simulation”, International Journal of Heat and Fluid Flow, Vol. 21, No. 3, 2000,pp. 252-263.

[9] Jameson, A., Schmidt, W.,Turkel, E. "Numerical solutions of the Euler equations by finite volume methods using RungeKutta time-stepping schemes". AIAA paper, 1259, 1981.

[10] Destarac D., "Far-field / Near-field Drag Balance and Applications of Drag Extraction in C.F.D." VKI Lecture Series, CFD Based Aircraft drag Prediction and Reduction, Hampton VA, 2003.

[11] van der Vooren, J., Destarac, D., "Drag/Thrust Analysis of Jet-propelled Transonic Transport Aircraft; Definition of Physical Drag Components," Aerospace Science \& Technology 8, 2004, pp. 545-556.

[12] Economon T. D., Palacios F., Copeland S. R., Lukaczyk T. W., and Alonso J. J., "SU2: An open- source suite for multiphysics simulation and design", AIAA Journal, 54(3):828-846, 2016. doi: 10.2514/1.J053813. 
[13] Chaput, A., Rizo-Patron, S., Vehicle Sketch Pad Structural Analysis Module Enhancements for Wing Design, 50th AIAA Aerospace Sciences Meeting, Nashville, TN, 2012, AIAA-2012-546.

[14] Hang Si. 2015. TetGen, a Delaunay-Based Quality Tetrahedral Mesh Generator. ACM Trans. on Mathematical Software. 41 (2), 2015. http://doi.acm.org/10.1145/2629697.

[15] Vanderplaats G. N., "Numerical Optimization Techniques for Engineering Design with Applications”, Mc Graw-Hill, 1984. [16] Vanderplaats G. N., "DOT user's manual”, version 4.20, 1995.

[17] Dakota: Design Analysis Kit for Optimization and Terascale Applications. Developed by SANDIA National Laboratories, Albuquerque, New Mexico, http://endo.sandia.gov/DAKOTA/software.html

[18] Wiart L., Atinault O., Grenon G., Paluch B., and Hue D.. "Development of NOVA aircraft configurations for large engine integration studies". In 33rd AIAA Applied Aerodynamics Conference, 2015. 


\section{Correction: Aerodynamic analysis and optimization of a boxwing architecture for commercial airplanes}

Author(s) Name: Marco Carini, Michael Méheut, Stylianos Kanellopoulos, Vittorio Cipolla, Karim Abu Salem. Author(s) Affiliations: ONERA The French Aerospace Lab, Meudon, F-92140, France; ONERA The French Aerospace Lab, Meudon, F-92140, France; ONERA The French Aerospace Lab, Meudon, F-92140, France; University of Pisa, Via G. Caruso 8, Pisa, Italy; University of Pisa, Via G. Caruso 8, Pisa, Italy.

\section{Correction Notice}

Results presented in Fig. 3 refer to an incidence of 0.0 degrees at the reference cruise conditions of $M=0.79$ and altitude $11 \mathrm{~km}$.

In Table 1, the total CD value reported in Table 1 corresponds to the near-field drag. The seeming discrepancy with the sum of the detailed drag components has therefore to be ascribed to the spurious drag contribution. Consistently with curves reported in Fig. 8, the value of L/D reported in the Table should be corrected accordingly by using the total far-field drag. An updated version of the table is reported below.

\begin{tabular}{ccccccccc}
\hline $\boldsymbol{A o \boldsymbol { A } ^ { \circ }}$ & $\boldsymbol{C L}$ & $\boldsymbol{C D}_{\boldsymbol{f f}}$ & $\boldsymbol{C D}_{\boldsymbol{f}}$ & $\boldsymbol{C D}_{v p}$ & $\boldsymbol{C D}_{\boldsymbol{w}}$ & $\boldsymbol{C D}_{\boldsymbol{i}}$ & $\boldsymbol{L} / \boldsymbol{D}$ & $\boldsymbol{e}$ \\
\hline $\mathbf{0 . 0}^{\circ}$ & 0.432 & 223.96 & 92.29 & 30.90 & 5.46 & 95.31 & 19.30 & 1.28 \\
$\mathbf{2 . 0}^{\circ}$ & 0.609 & 406.82 & 88.13 & 81.89 & 40.96 & 195.84 & 14.97 & 1.24 \\
\hline
\end{tabular}

At page 11, in the fifth sentence of the first paragraph, there is a typo which leads to a misleading conclusion: 'except for section 7 ' has to be replaced by 'except for section 8 '.

The total $\mathrm{CD}$ value reported in Table 4 correctly corresponds to the far-field drag value. Results refer to cruise conditions.

Unfortunately CFD results illustrated in Fig. 11, 12 and 13 concerning the UNIPI configuration were affected by some minor convergence issues which have not been detected before the paper submission. We apologize for this. Comments and conclusions reported in the paper are still supported by fully checked results which are illustrated below.

a)

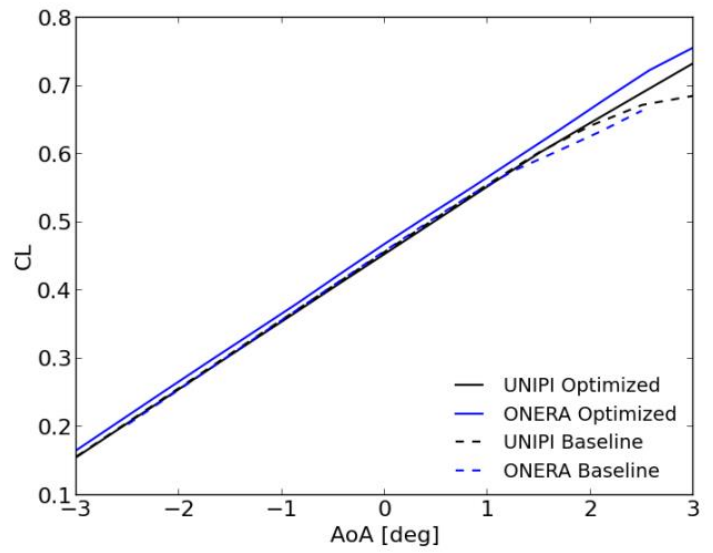

b)

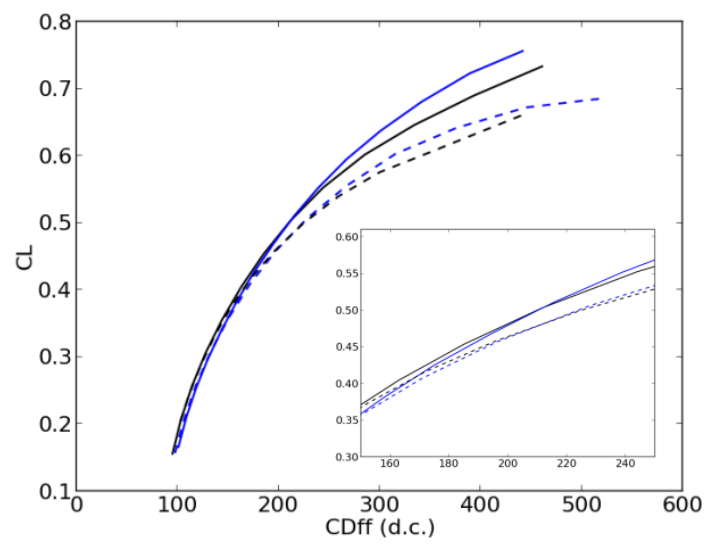

Fig. 1. RANS assessment of UNIPI and ONERA Euler optimized configurations w.r.t. to corresponding baselines. (a) Lift curve. (b) Far-field drag polar curve. 


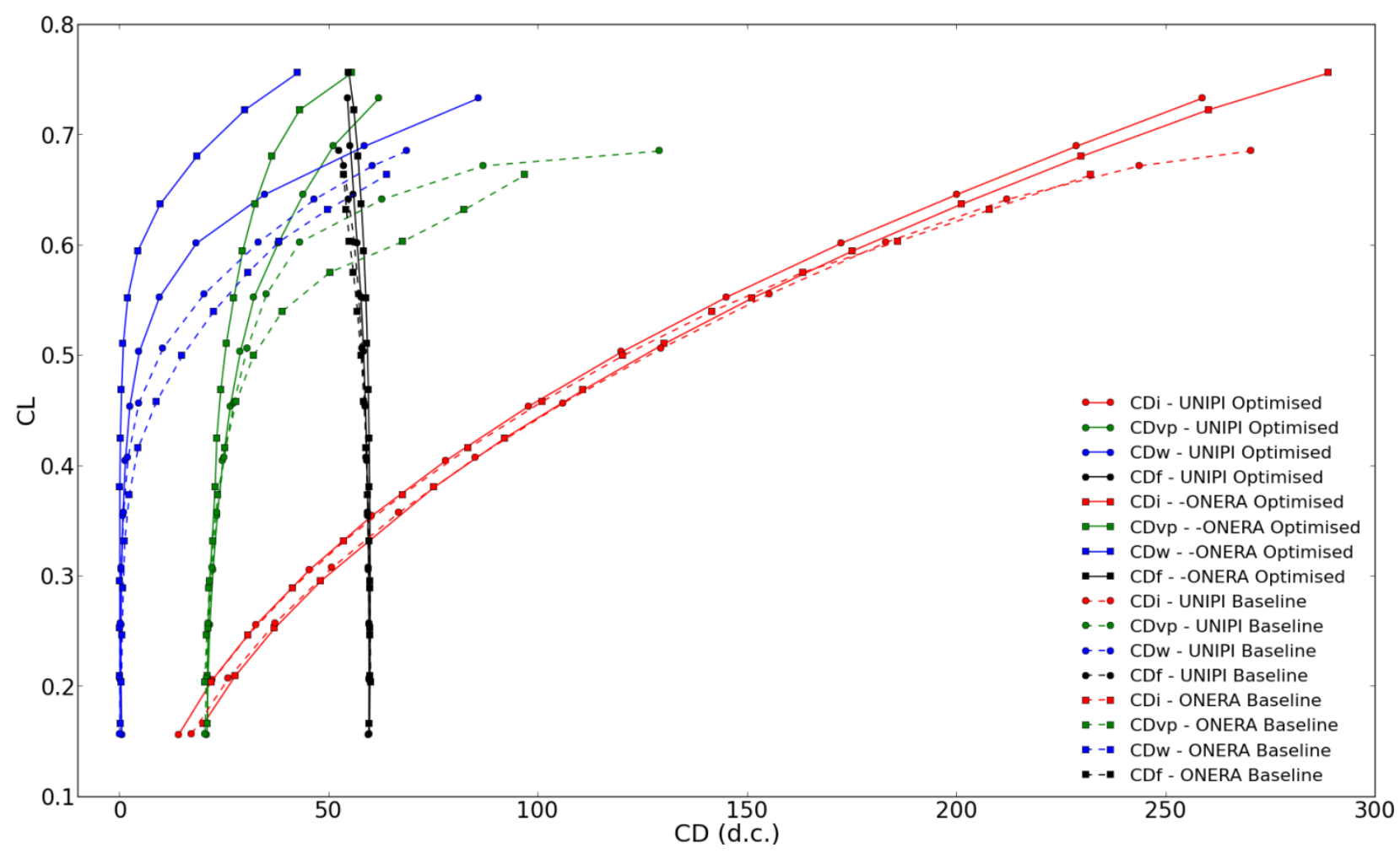

Fig. 2. RANS assessment of UNIPI and ONERA Euler optimized configurations w.r.t. to corresponding baselines: far-field drag breakdown. Optimised configuration refer to the simultaneous optimisation of both twist and camber design variables.

a)

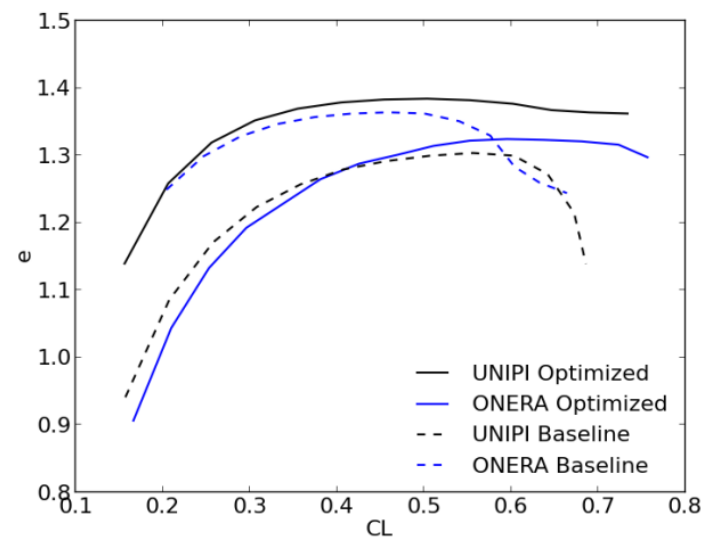

b)

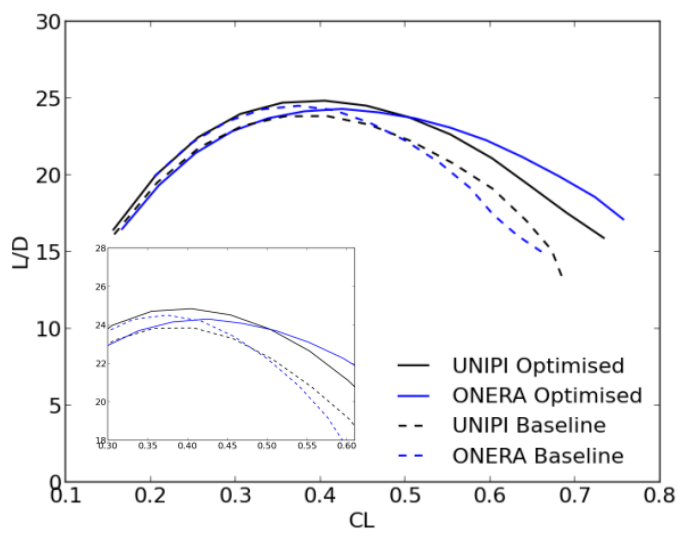

Fig. 3. RANS assessment of UNIPI and ONERA Euler optimized configurations w.r.t. to corresponding baselines: (a) Oswald efficiency. (b) Aerodynamic efficiency. Optimised configuration refer to the simultaneous optimisation of both twist and camber design variables. 
According to the corrected results illustrated in Fig. 13(b) just above, the last sentence in the first paragraph of page 14:

"Comparison in terms of total aerodynamic efficiency is then reported in Fig. 13(b) assessing the superior improvement achieved by the ONERA optimized design for $C L>0.4$."

has to be modified as follows:

"Comparison in terms of total aerodynamic efficiency is then reported in Fig. 13(b) assessing the superior improvement achieved by the ONERA optimized design for $C L>0.5$."

The CL threshold value has to be also corrected in the concluding remarks on page 15, second paragraph, with:

"The ONERA design achieves the best performance in terms aerodynamic efficiency in the range of CL $>0.5$ ".

in place of :

"The ONERA design achieves the best performance in terms aerodynamic efficiency in the range of CL>0.4".

The results presented in Fig. 14 and Fig. 15 for the ONERA configuration are here integrated with those corresponding to the UNIPI configuration.

a)

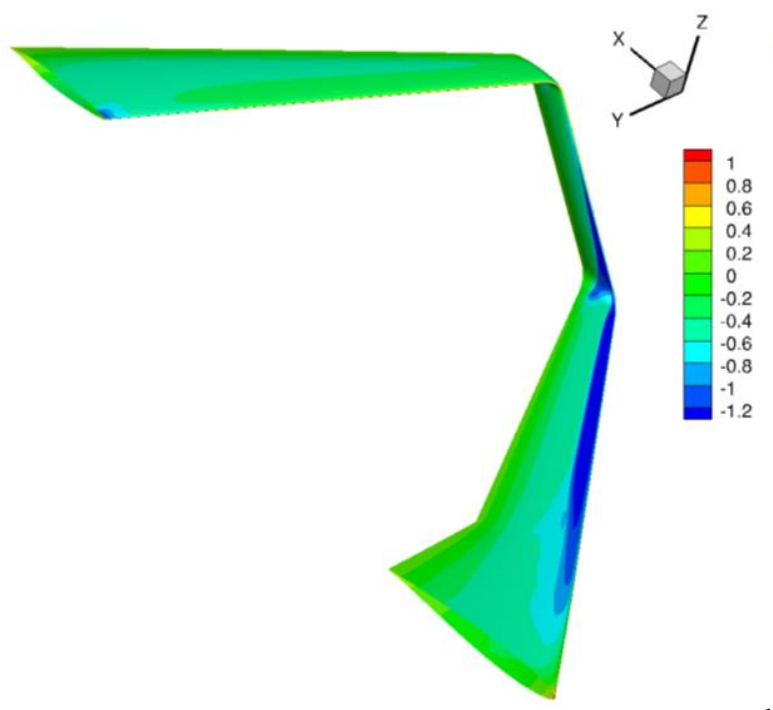

c)

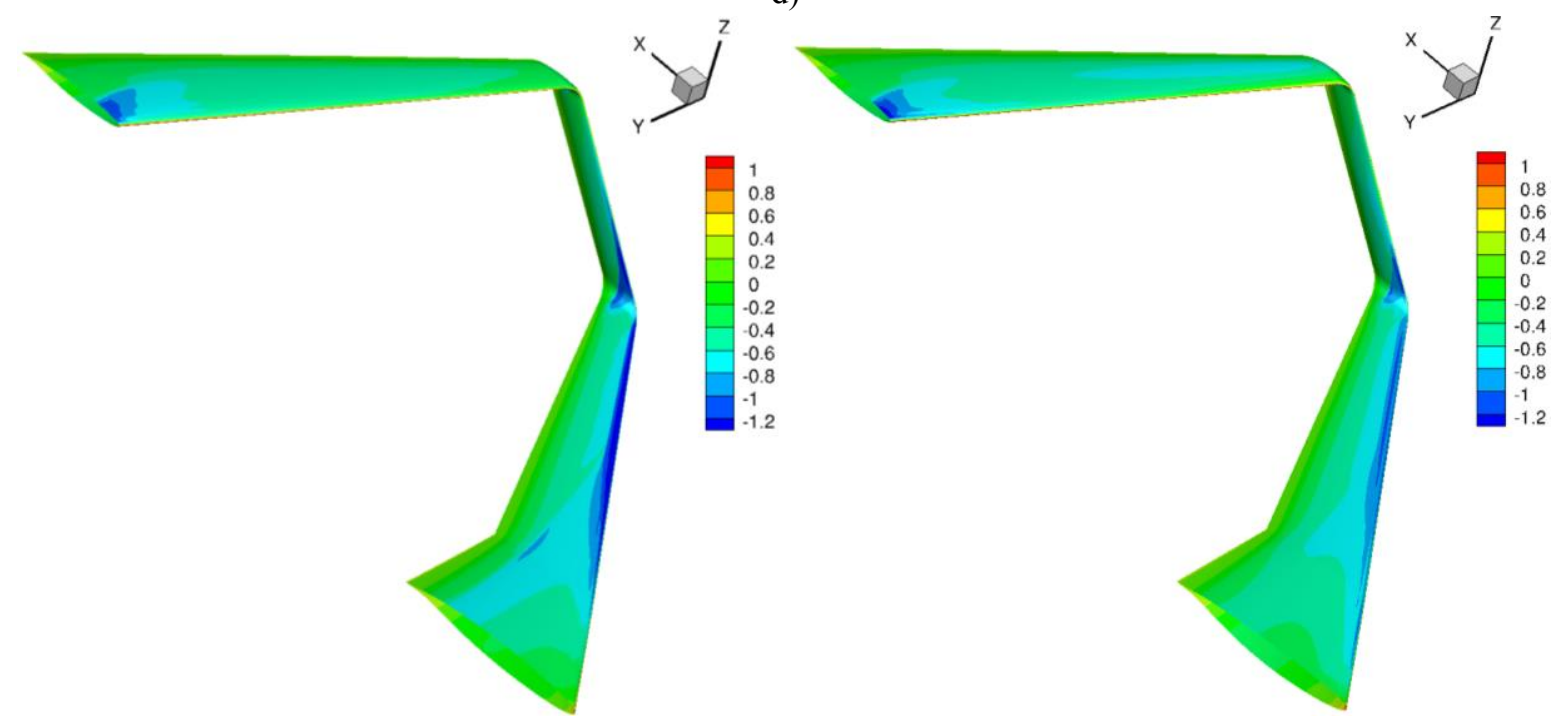

b)

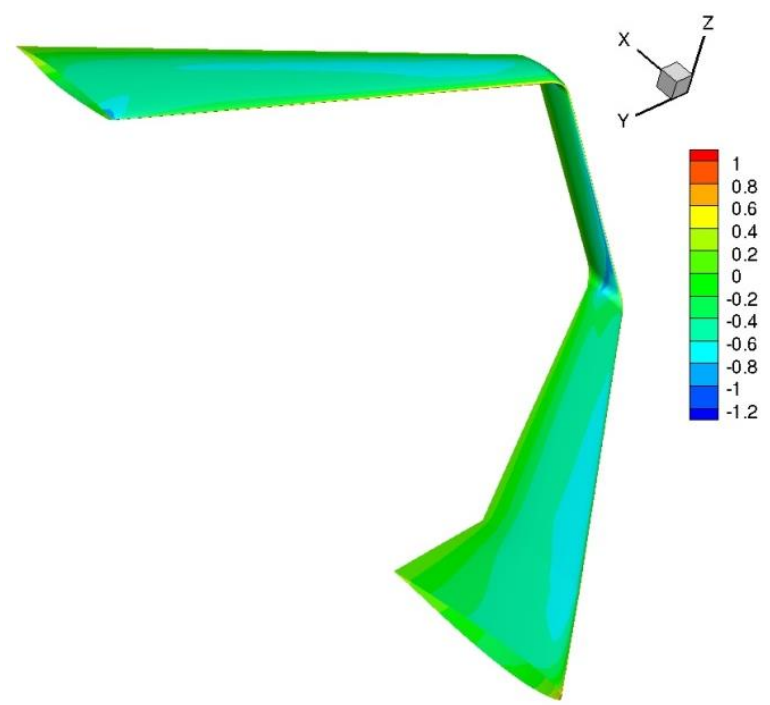

d)

Fig. 4. RANS assessment of ONERA and UNIPI Euler optimized configurations w.r.t. to corresponding baselines: comparison of pressure coefficient $(C p)$ distributions. (a) ONERA Baseline at $C L=0.458$. (b) ONERA Optimized at $C L=0.469$. (c) UNIPI Baseline at $C L=0.457$. (d) UNIPI Optimized at $C L=0.454$. Optimised configuration refer to the simultaneous optimisation of both twist and camber design variables. 
a)

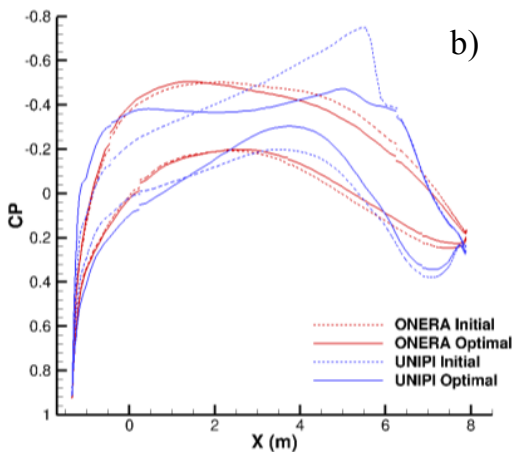

d)

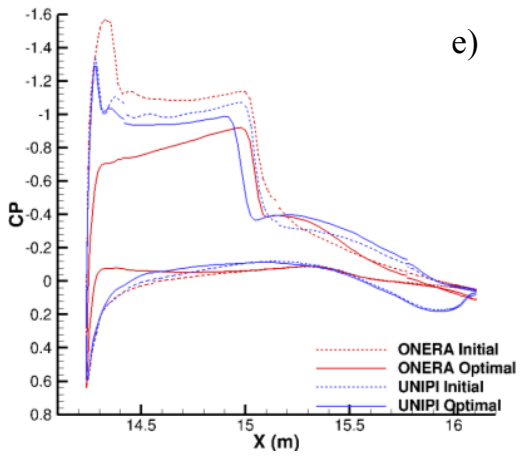

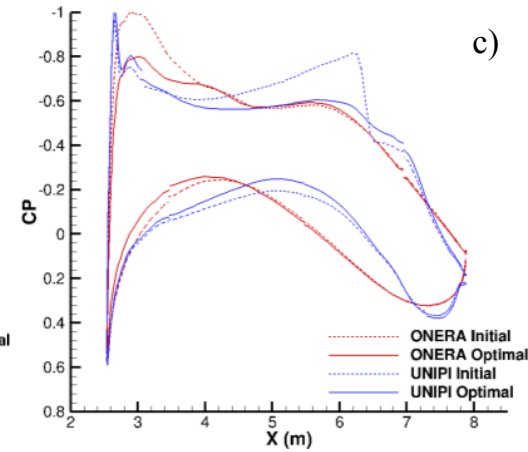

c)

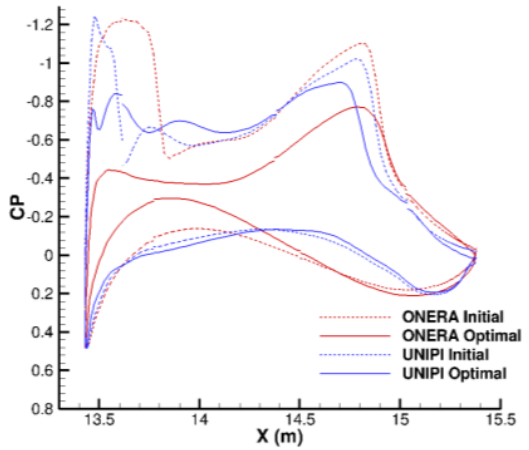

f)
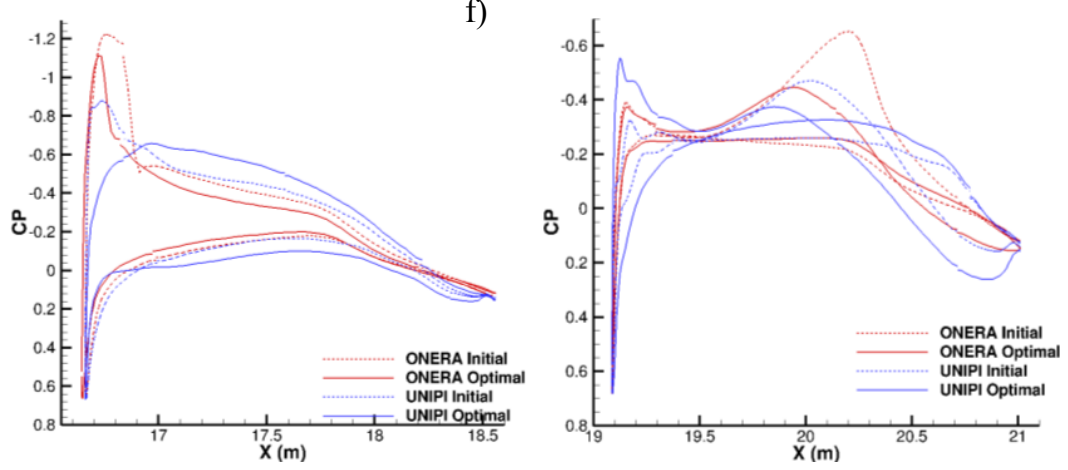

g)

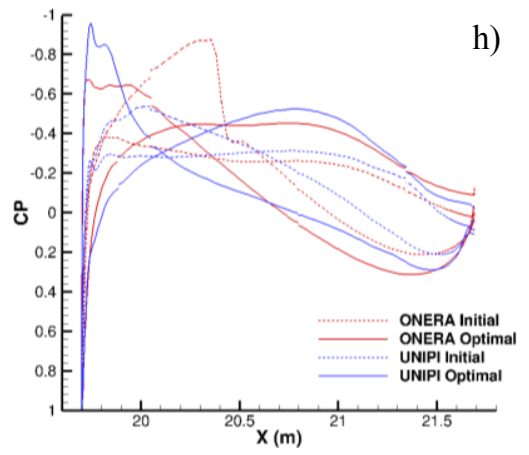

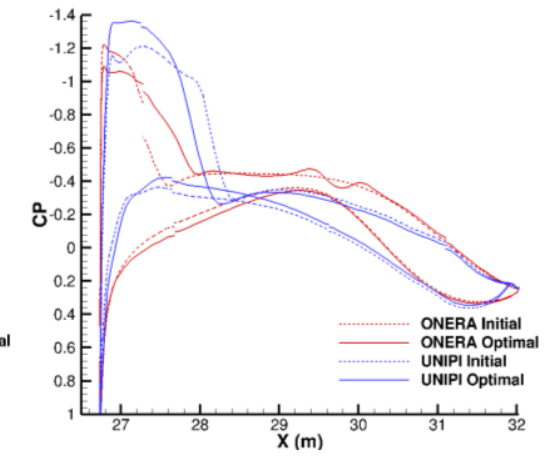

Fig. 5. RANS assessment of ONERA and UNIPI optimized configurations w.r.t. to corresponding baselines. Comparison of pressure coefficient $(C p)$ distributions at selected sections from Fig. 14: (a) section 1; (b) section 2; (c) section 3; (d) section 4; (e) section 5; (f) section 6; (g) section 7; (h) section 8 .

Given the above supplementary results, the same comments provided in the second paragraph of page 14 also apply to the UNIPI configuration. 